\title{
Progress in Doping Semiconductor Nanowires During Growth
}

Shadi A. Dayeh, ${ }^{1,2,3}$ Renjie Chen, ${ }^{1}$ Yun Goo Ro, ${ }^{1}$ and Joonseop Sim ${ }^{1}$

\begin{abstract}
The anisotropic growth of one-dimensional or filamental crystals in the form of microwires and nanowires constitutes a rich domain of epitaxy and newly enabled applications at different length and size scales. Significant progress has been accomplished in controlling the growth, morphology, and properties of semiconductor nanowires and consequently their device level performance. The objective of this review is two-fold: to highlight progress up to date in nanowire doping and to discuss the remaining fundamental challenges. We focus on the most common semiconductor nanowire growth mechanism, the vapor-liquid-solid growth, and the perturbation of its kinetic and thermodynamic aspects with the introduction of dopants. We survey the origins of dopant gradients in nanowire growth and summarize quantification techniques for dopants and free-carrier concentrations. We analyze the morphological changes due to dopants and the influence of growth droplet seeds on composition and morphology and review growth aspects and alternatives that can mitigate these effects. We then summarize some of the remaining issues pertaining to dopant control in nanowires.
\end{abstract}

\section{An Overview of Nanowire Growth}

The visionary works of Wagner and Ellis in the 1960s have predicted the evolution of microwire growth and their doping when they stated "Controlled growth can be obtained through appropriate use of impurities in patterns of films on substrate surfaces and on single-crystal seeds of many substrates. P-N junctions and heterojunctions can be made." In the years that followed, $\mathrm{Si},{ }^{2} \mathrm{Ge}$, ${ }^{3}$ and their axial heterostructures, ${ }^{4}$ as well as compound semiconductors such as $\mathrm{GaAs},{ }^{5} \mathrm{GaP},{ }^{6} \mathrm{InAs},{ }^{7}$ and their alloys of InGaAs, GaAsP, ${ }^{8}$ have already been realized in the period of 1960-1980. The first device on a 1D nanowire (NW) was fabricated in 1991 by Haraguchi et al. who formed p-n junctions from GaAs whiskers grown using selective area metal-organic chemical vapor deposition. ${ }^{9}$ A global renaissance in NW growth occurred in 1998 when A. M. Morales and C. M. Lieber utilized VLS to prepare Si and Ge NWs with diameters of $3-20 \mathrm{~nm}$ and lengths of $1-30 \mu \mathrm{m} .{ }^{10}$ This led to fascinating progress in the formation of axial ${ }^{11,12,13}$ and radial ${ }^{14}$ heterostructure growth with efficient size $^{15}$ and alloy ${ }^{16}$ bandgap engineering enabling new frontiers in crystal engineering, ${ }^{17,18,19}$ and applications in diverse areas such as electronics, ${ }^{20}$ photonics,${ }^{21}$ thermoelectrics, ${ }^{22}$ chemical sensing, ${ }^{23}$ biosensors, ${ }^{24}$ and biostimulants, ${ }^{25}$ to name a few. 
Semiconductor NWs can be synthesized using a variety of growth techniques including organo-metallic vapor phase epitaxy (OMVPE), ${ }^{26,27,28}$ selective area OMVPE (SA-OMVPE), ${ }^{29}$ molecular ${ }^{30}$ and chemical ${ }^{31}$ beam epitaxy, wafer annealing, ${ }^{32}$ chemical vapor deposition (CVD), ${ }^{33}$ laser ablation, ${ }^{34,35}$ and low temperature solution methods. ${ }^{36}$ In the presence of foreign catalytic metal particles that seed NW growth, their evolution is interpreted to occur via the Vapor-Liquid-Solid $(\mathrm{VLS})^{1}$ growth mechanism. In the absence of foreign metal particles, their growth is interpreted via group-III catalyzed VLS growth,,37 oxide-assisted growth, ${ }^{35}$ ligand-aided solution-solid (LSS) growth, ${ }^{38}$ reactive $\mathrm{Si}$-assisted growth, ${ }^{39}$ and dislocation-driven growth. ${ }^{40}$

The most applicable growth mechanism for semiconductor NWs is the VLS growth mechanism that is briefly overviewed in Figure 1a-c. It is centered around the formation of a supersaturated eutectic droplet that facilitates the NW growth in a layer-by-layer fashion. The prerequisite conditions for epitaxial NW growth include the formation of liquid solution between the metal seed and the material to be grown (Figure 1a) and must usually have a large contact angle $\left(95^{\circ}-\right.$ $120^{\circ}$ ) with the growth substrate to enable its rise above the surface. The chemical reactions, whether homogeneous or heterogeneous, should be thermodynamically possible but not favored kinetically so that the catalytic and adsorption properties of the liquid growth seed become effective in precursor decomposition and incorporation (Figure 1b) and consequently in the one-dimensional NW growth. ${ }^{3}$ In addition, high supersaturations in the growth seed are required especially in the initial stages of growth to enable its rise above the substrate surface, which must be oxide free to enable the vertical epitaxial growth. In steady state, the rate of adatom incorporation at the liquid-solid interface (Figure $1 \mathrm{~b}, \mathrm{R}_{3}$ ) is equal to the rate of precursor decomposition and incorporation at the vapor-liquid interface (Figure $1 \mathrm{~b}, \mathrm{R}_{1}$ ). The diffusion through the liquid particle (Figure $1 \mathrm{~b}, \mathrm{R}_{2}$ ) is not rate limiting. The above requirements determine the temperature ranges over which NW growth is preferred, which are typically $100-200{ }^{\circ} \mathrm{C}$ lower than those used in thin film growth. Within the NW growth temperature window, low temperatures may not allow the formation of the liquid growth seed alloy and oxide desorption, whereas high temperatures increase the material solubility in the liquid nanoparticle (NP) and reduce its supersaturation and consequently the NW nucleation and growth rates. Figure 2 shows a scanning electron microscope (SEM) image of an array of Ge NWs with 70nm diameter grown epitaxially by CVD on a Ge(111) substrate from a 20nm thick pre-patterned Au disc by electron beam lithography. The experimental growth regimes for Ge and Si NWs are highlighted under the liquidius line of the bulk Au-Si and Au-Ge binary phase diagram in Figure 1d.

The driving force for growth, supersaturation $\Delta \mu=\mu_{v}-\mu_{s} \cong \mu_{l}-\mu_{s}$ is the change in the chemical potential during the phase transformation from the liquid droplet to the solid NW. If $\Phi$ is the thermodynamic potential, and $\mathrm{N}$ is the number of atoms in the crystal during growth, then 
$\Delta \mu=\delta \phi / \delta N$. One can compute the changes in $\delta \phi$ in terms of the variation of the system free energy $-\pi r^{2} \delta L \Delta \mu_{0} / \Omega$ where $r$ is the NW radius, $\delta L$ is the incremental change in the NW length, assuming a cylindrical NW, $\Omega$ is the atomic volume of the NW material in the growth seed, and $\Delta \mu_{0}$ is the supersaturation without surface considerations, i.e. in the bulk limit. For every $\delta L$ in the NW, there is an increase in the surface free energy $2 \pi r \delta L \alpha_{v s}$ where $\alpha_{v s}$ is the NW surface energy density. Therefore, $\delta \phi=-\pi r^{2} \delta L \Delta \mu_{0} / \Omega+2 \pi r \delta L \alpha_{v s}$ and with $\delta N=\pi r^{2} \delta L / \Omega$, one can therefore write:

$$
\Delta \mu=\Delta \mu_{0}-\frac{4 \Omega \alpha_{v s}}{d} \quad \text { or } \quad \frac{\Delta \mu}{k T}=\frac{\Delta \mu_{0}}{k T}-\frac{4 \Omega \alpha_{v s}}{k T} \frac{1}{d}
$$

which is pressure and temperature dependent $\left(\Delta_{\mu_{0}}\right)$ and inversely proportional to diameter $d$. In other words, one can utilize the Gibbs-Thompson effect for the rise of partial pressure at small sizes according to 41

$$
P_{0}=P_{\infty} e^{\frac{4 \Omega \alpha_{V L}}{d k T}} \cong P_{\infty} e^{\frac{4 \Omega \alpha_{V S}}{d k T}}
$$

to reach equation (1) by utilizing $\Delta \mu=k T \ln \left(P_{\text {in }} / P_{0}\right)$ where $P_{\infty}$ and $P_{0}$ are the equilibrium partial pressures of the grown material in bulk and NW forms, respectively, and $P_{i n}$ is the gas precursor input partial pressure.

If the adatoms have negligible diffusion along the NW sidewalls, the axial growth velocity can be empirically related to supersaturation with $v=b(\Delta \mu / k T)^{2}$, where $b$ is a temperature independent kinetic coefficient of crystallization. ${ }^{42,43}$ Thus, using equation 1, one can write an equation of the growth velocity as a function of supersaturation as

$$
\sqrt{v}=\sqrt{b} \frac{\Delta \mu_{0}}{k T}-\sqrt{b} \frac{4 \Omega \alpha_{v s}}{k T} \frac{1}{d}
$$

from which $L=t \cdot b \cdot\left(\Delta \mu_{0} / k T-4 \Omega \alpha_{v s} / k T d\right)^{2}$ can be obtained.

Using these equations, one can summarize the thermodynamic dependencies on the growth in Figure 1e, which shows enhanced supersaturations and normalized growth rates for higher overdrive pressures and lower temperatures. 
One can also deduce from equation (2) that the phase diagram in Figure 1d does not apply to NW growth where for a given temperature, the equilibrium compositions are higher in NWs at smaller $d$ compared to their bulk counterparts. Using in-situ transmission electron microscopy (TEM) heating experiments, one can quantify the equilibrium composition of the growth seed in the absence of any input precursor. ${ }^{42}$ Indeed, the experimental measured data follow qualitatively equation (3) or its composition equivalent by invoking Henry's law to obtain,

$C_{0}=C_{\infty} \exp \left(\kappa 4 \Omega \alpha_{v s} / d k T\right)$

where $\varkappa$ is a fit parameter that accounts for the triple-phase curvature changes with temperature. This force-balance at the triple-phase boundary is governed by $\sigma_{l} \cos \beta=\sigma_{s} \cos \alpha-\sigma_{\text {ss }}-\tau / r_{0}$ where $\beta$ is the contact angle at the solid-liquid interface, $\alpha$ is the angle between the liquid-solid interface and the sidewall of the nanostructure $\left(\alpha=90^{\circ}\right.$ for NW), $\sigma_{l}{ }^{\prime} \sigma_{s}$, and $\sigma_{l s}$ are the liquid-vapor, solid-vapor, and liquid-solid surface energy densities, $\tau$ is the droplet surface line tension, and $r_{0}$ is the radius of its contact cross-section or the NW radius. $\tau / r_{0}$ is negligible compared to the other terms. ${ }^{43}$ In the Au-Ge material system, $\sigma_{l s}\left(460{ }^{\circ} \mathrm{C}\right)=0.319 \mathrm{~J} / \mathrm{m}^{2}$ and $\sigma_{l s}\left(584^{\circ} \mathrm{C}\right)=0.211 \mathrm{~J} / \mathrm{m}^{2}$ while $\sigma_{l}\left(460^{\circ} \mathrm{C}\right)=0.798 \mathrm{~J} / \mathrm{m}^{2}$ and $\sigma_{l}\left(584^{\circ} \mathrm{C}\right)=0.765 \mathrm{~J} / \mathrm{m}^{2} .{ }^{44}$ This indicates a smaller contact angles with higher temperatures (higher compositions) which in this case changes from $113.56^{\circ}$ at $460{ }^{\circ} \mathrm{C}$ to $106^{\circ}$ at $584{ }^{\circ} \mathrm{C}$. This trend is qualitatively in agreement with what is observed experimentally in Ge NWs, ${ }^{42}$ where the composition is fit using equation (4) in Figure 1f and the NW phase diagram relative to bulk is constructed in Figure 1g. It is clear that the liquidius line is suppressed compared to that of bulk. ${ }^{45}$ Liquid Au-Ge particles have been observed at temperatures that are $120^{\circ} \mathrm{C}$ lower than the bulk eutectic temperature, 46 signifying these effects and the applicability of the VLS mechanism at deep sub-eutectic temperatures. 

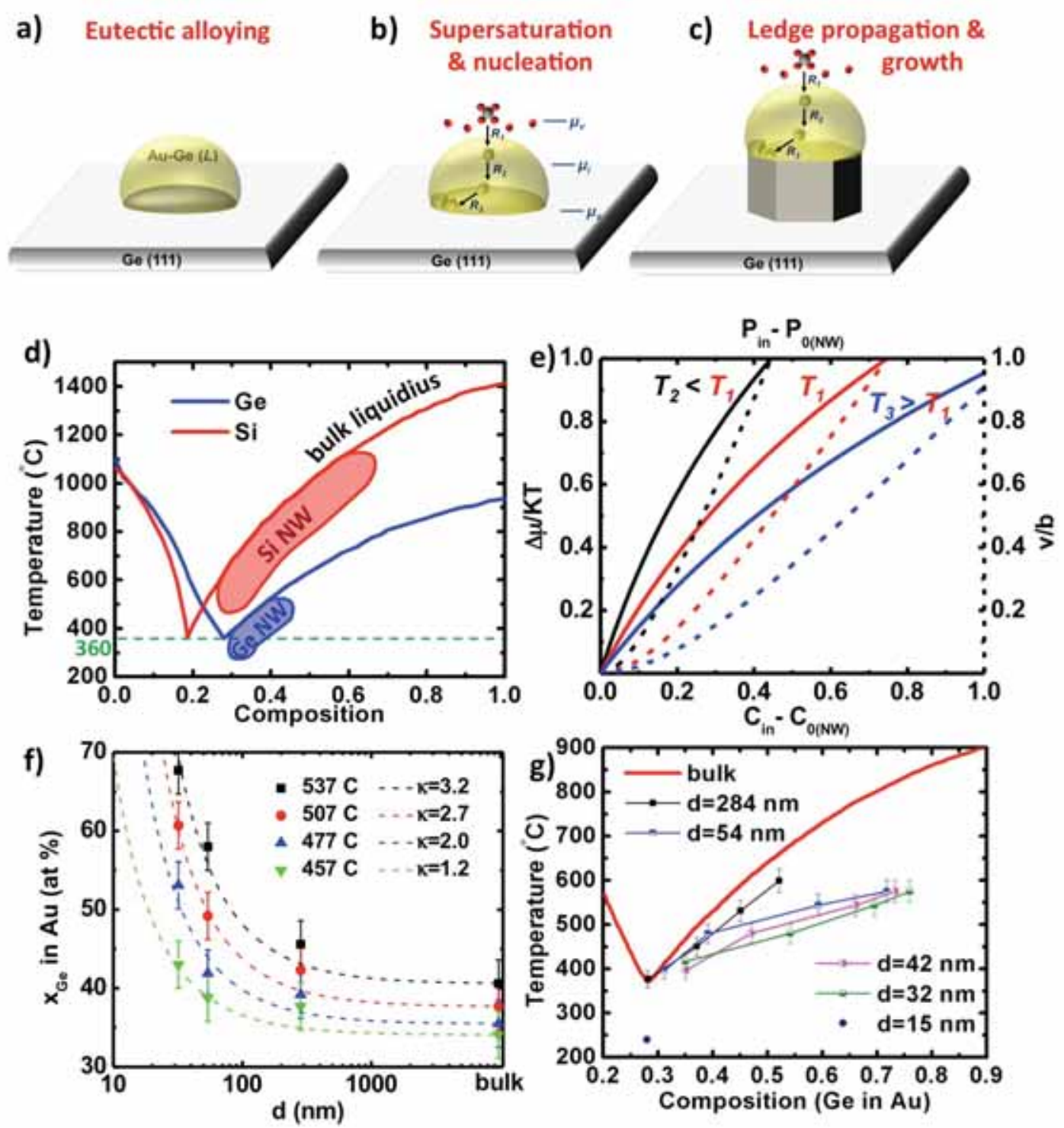

Figure 1: (a) - (c) Schematic illustration for the VLS NW growth. (d) Bulk phase diagram for the Ge$\mathrm{Au}$ and $\mathrm{Si}-\mathrm{Au}$ and the temperature windows for Ge and Si NW growth, (e) Supersaturation and thermodynamically limited growth rate (normalized to kinetic coefficient) as a function of overpressure for different temperatures. $P_{i n}$ and $C_{i n}$ are the input partial pressure and concentration, respectively, that are simply related with a proportionality constant using Henry's law. f) Measured Ge composition in $\mathrm{Au}$ seeds atop pre-grown Ge NWs in-situ TEM. Panel (f) is Copyright American Chemical Society. Reproduced with permission from ref. [47]. (g) Suppression of the liquidius line in different diameter Ge NWs. Reproduced after Sutter et al. from ref. [42]. $15 \mathrm{~nm}$ diameter data point from Gamalski et al. ref. [46]. Copyright American Chemical Society. 


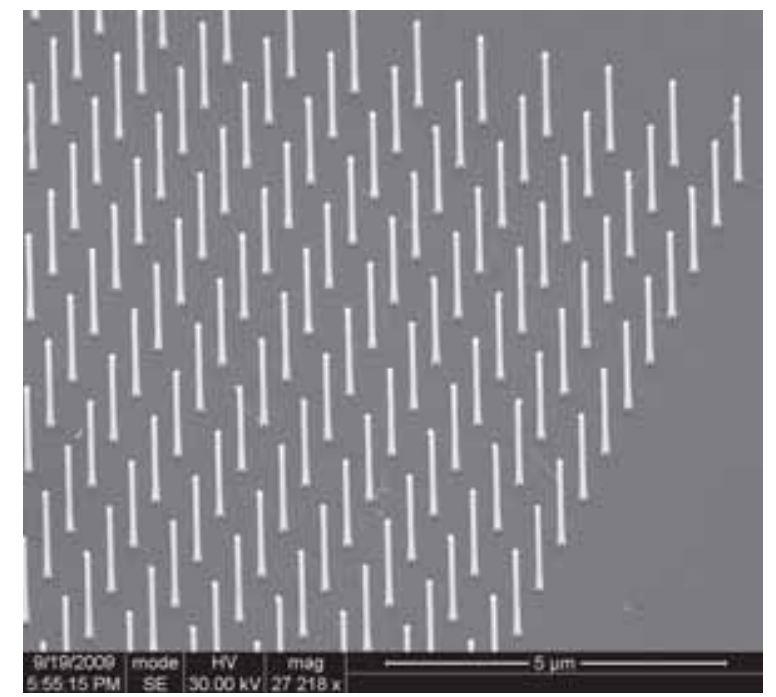

Figure 2: SEM image of an array of Ge NWs $(d=70 \mathrm{~nm})$ grown epitaxially on a Ge(111) substrate from a Au-Ge alloy particle.

\section{Influence of Doping on Nanowire Growth Rate}

$\mathrm{N}$-type and p-type doping can be achieved in-situ by introducing phosphine $\left(\mathrm{PH}_{3}\right)$ and diborane $\left(\mathrm{B}_{2} \mathrm{H}_{6}\right)$ gas precursors during $\mathrm{Si}$ and $\mathrm{Ge} \mathrm{NW}$ growth. These doping precursors decompose and dissolve in the liquid growth seed and incorporate into the NW body as will be further elaborated in section III. ${ }^{48}$ The introduction of dopant precursors influences the NW growth rate depending on the dopant species or the dopant-to-Si gas precursor ratios. The primary effects are due to (1) changes in supersaturation with dopant introduction and/or (2) changes to the NW surface energy due to the dopant precursor or its decomposed by-products. ${ }^{49}$ It has been reported that the Si NW nucleation could be completely inhibited with high phosphine-to-silane ratios, ${ }^{50}$ and similar results were also observed in arsine $\left(\mathrm{AsH}_{3}\right)^{51}$ or trimethylantimony (TMSb) ${ }^{52}$ doped Si NWs.

We have performed systematic studies of the impact of dopants on the growth rate of Ge NWs (Figure 3 and Figure 4). ${ }^{47}$ Figure 3 shows a sequence of SEM images taken from the center of undoped or Boron-doped NW arrays grown under similar growth conditions (temperature, time, and total chamber pressure) and displaying a size- and doping-dependent lengths. In the presence of $\mathrm{PH}_{3}$ and $\mathrm{B}_{2} \mathrm{H}_{6}$, the resultant NW lengths are shorter than that of the undoped NWs (Figure 4a). Naturally, for the same total pressure, the introduction of dopant precursors reduce the partial pressure of $\mathrm{GeH}_{4}$ responsible for NW growth, and therefore, the growth rate is unsurprisingly reduced. To account for the partial pressure variation, Figure $4 \mathrm{~b}$ shows the pressure-normalized growth rate, $v / P_{i n}=L /\left(t \cdot P_{i n}\right)$, as a function of $\mathrm{NW}$ diameter. In the presence of $\mathrm{B}_{2} \mathrm{H}_{6}$ the normalized growth rate decreased slightly, while the presence of $\mathrm{PH}_{3}$ showed more striking behaviors. We found that $V / P_{\text {in }}$ is lower than that of undoped NWs for $\mathrm{d} \geq 20 \mathrm{~nm}$ (progressively changing from a $\sim 1 \%$ 
reduction at $\mathrm{d}=20 \mathrm{~nm}$ to $\sim 6.5 \%$ at $\mathrm{d}=80 \mathrm{~nm}$ ), while higher $v / P_{\text {in }}$ are obtained for $\mathrm{d}<20 \mathrm{~nm}$ (again changing from a $\sim 2 \%$ increase at $\mathrm{d}=15 \mathrm{~nm}$ to $\sim 22 \%$ at $\mathrm{d}=7.5 \mathrm{~nm}$ ). To better understand this

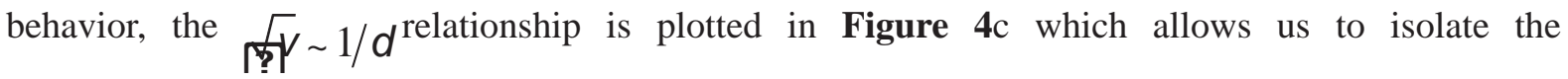
correspondent growth parameters such as supersaturation, surface energy density, and kinetic factor $b$ using equation (4). The result is listed in Table 1. The normalized supersaturation to partial pressure ratio was then plotted according to equation (3) as shown in Figure 4d. We observed that with both $\mathrm{B}_{2} \mathrm{H}_{6}$ and $\mathrm{PH}_{3}$ flow, the normalized supersaturation decreased for all studied diameters. Therefore, the interesting behavior with $\mathrm{PH}_{3}$ is attributed to the change in surface energy density $\alpha_{v S}$ as shown in

Table 1. Undoped and $\mathrm{B}_{2} \mathrm{H}_{6}$ doped Ge NW growths showed similar $\alpha_{v s}$, while $\mathrm{PH}_{3}$ doping reduced the $\alpha_{v S}$ contributing to the accelerated growth rate at smaller diameters $(\mathrm{d}<20 \mathrm{~nm}$ ). This further indicates that introduction of P-dopant has prominent effect on the NW sidewall surface, which is in general agreement with the incorporation of high concentrations of P into NW sidewalls. ${ }^{53}$

\section{Undoped}

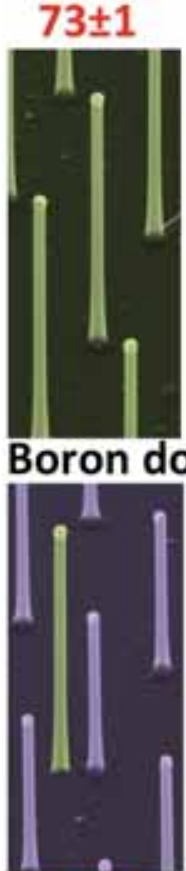

$76 \pm 1$
$68 \pm 3$
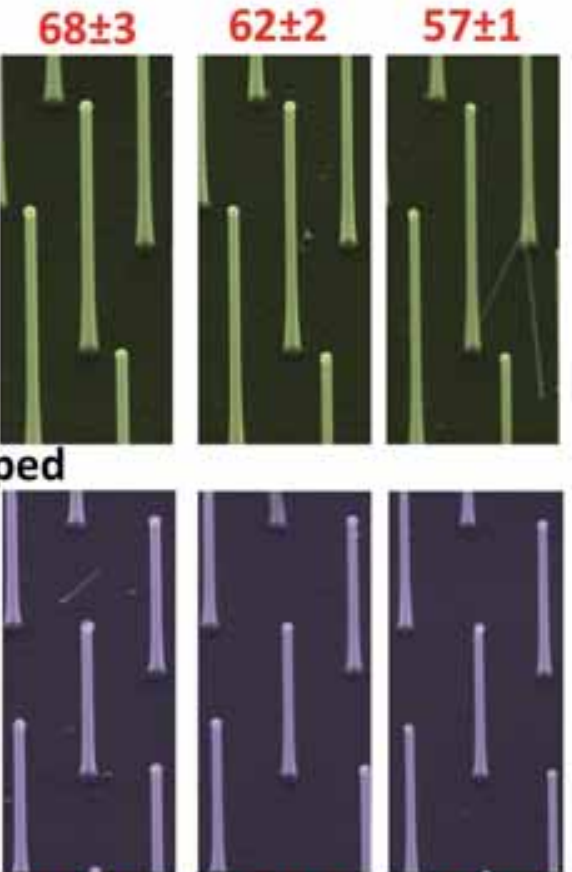

$70 \pm 1$

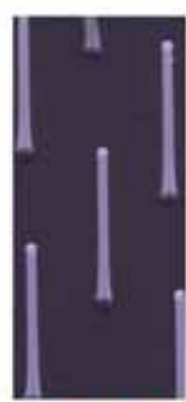

$64 \pm 1$

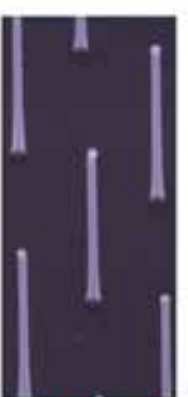

$58 \pm 1$
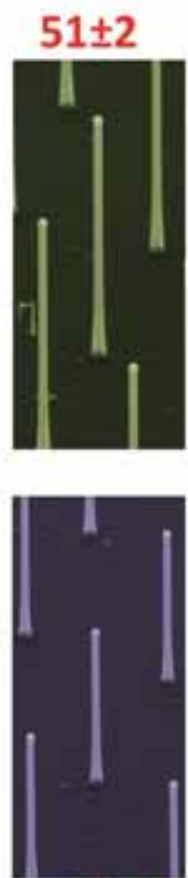

$53 \pm 1$

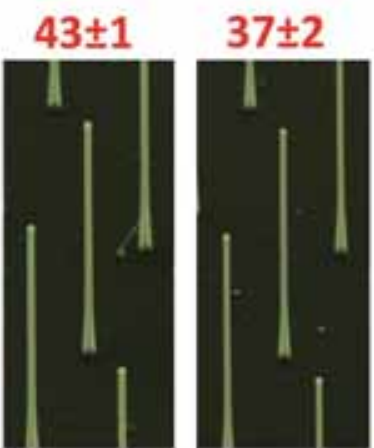

$29 \pm 1$
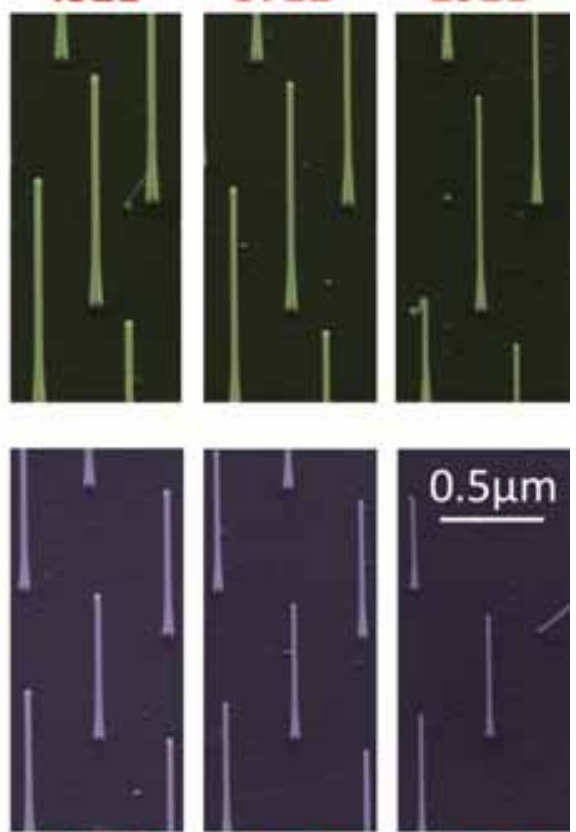

$44 \pm 1$

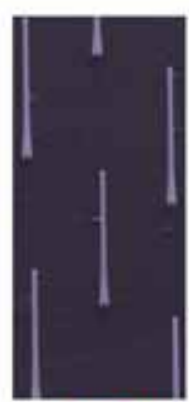

$37 \pm 1$

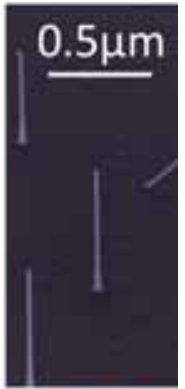

$18 \pm 1$

Figure 3: Colorized SEM images taken at the center of NW arrays with a constant diameter indicated on top of the top row of undoped NWs, and at the bottom of the lower Boron doped array (unit is nm), showing a diameter dependent growth rate evident for smaller diameter NWs. Both sets of arrays were grown under the same growth temperature and time using $\mathrm{GeH}_{4}$ and the B-doped NWs were grown with a $\mathrm{B}_{2} \mathrm{H}_{6}$ flow (see text for details). Superimposed on the lower left image is an undoped NW (green) showing reduced length for the B-doped NWs. 
(a)
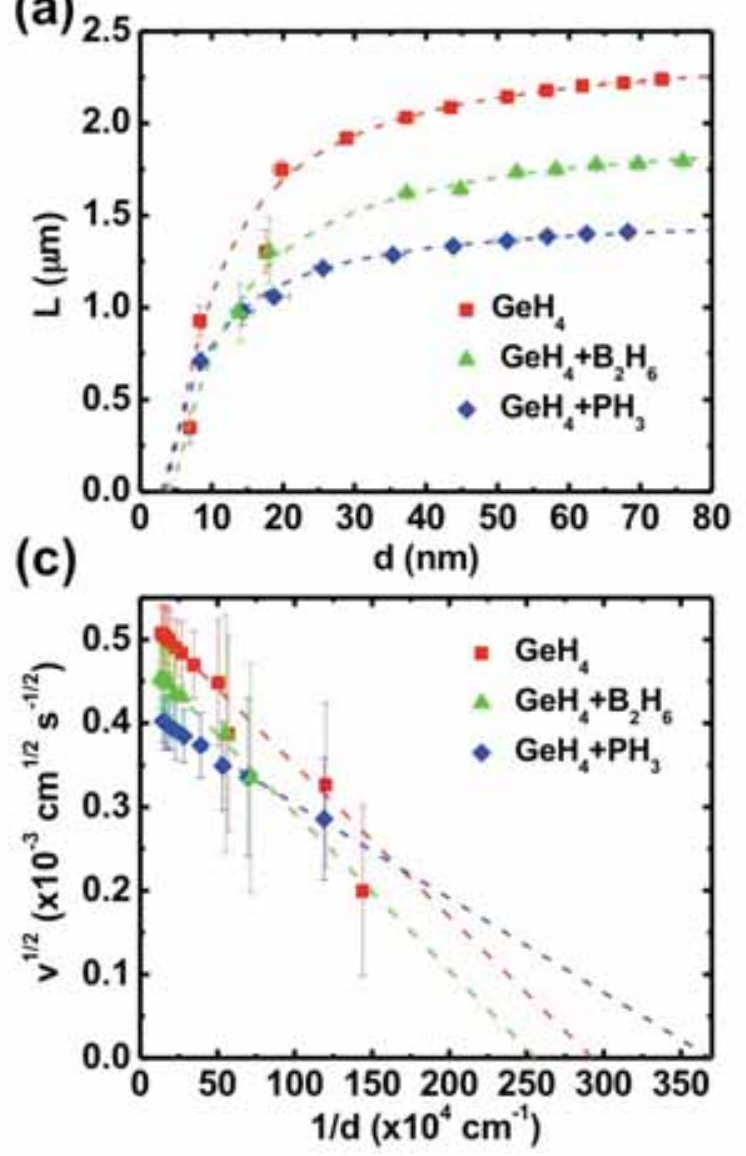

(b)
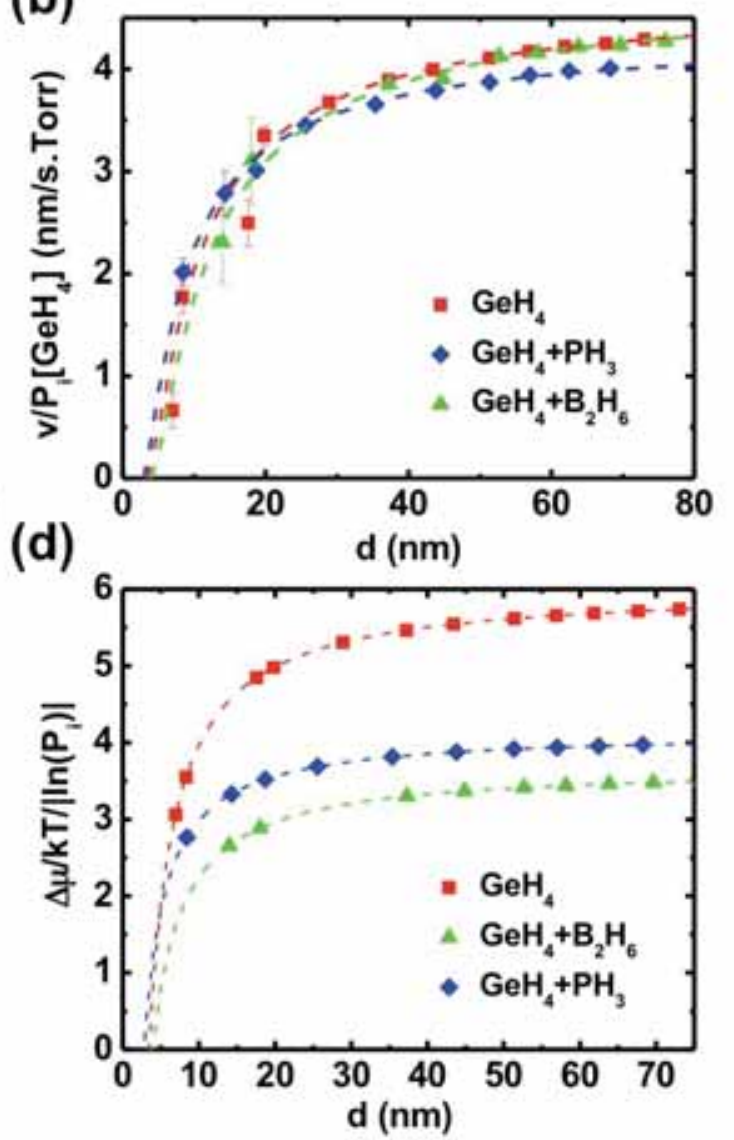

Figure 4: Plots of Ge NW growth rate with and without doping. (a) NW length as function of diameter for the same growth time. (b) Normalized growth rate by Ge partial pressure as function of NW diameter. (c) Square root of growth rate as function of inverse diameter. (d) Normalized supersaturation as a function of NW diameter. Reprinted with permission from the American Chemical Society, copyright 2010, Ref. [47].

Table 1: Calculated critical diameters (cut-off diameter where growth is completely suppressed, that is $v=0$ case in equation 4), pressure-normalized supersaturation values and kinetic coefficients. Reprinted with permission from the American Chemical Society, copyright 2010, Ref. [47].

\begin{tabular}{c|c|c|c}
\hline Precursors & $d_{c}(\mathrm{~nm})$ & $\Delta \mu_{0} / k T / \ln \left(P_{i n}\right)$ & $b^{1 / 2} \alpha_{v S}\left(10^{-8} \mathrm{~J} / \mathrm{cm}^{5 / 2} / \mathrm{s}^{1 / 2}\right)$ \\
\hline $\mathrm{GeH}_{4}$ & 3.4 & 6.0 & 1.5 \\
\hline $\mathrm{GeH}_{4}+\mathrm{B}_{2} \mathrm{H}_{6}$ & 3.9 & 4.1 & 1.6 \\
\hline $\mathrm{GeH}_{4}+\mathrm{PH}_{3}$ & 2.8 & 3.7 & 0.98 \\
\hline
\end{tabular}

\section{Dopant Incorporation and Gradients During Nanowire Growth}

To understand dopant incorporation, we should consider the nucleation mechanism in the VLS growth. While attempting to explain the origins of formation of Wurtzite crystal NWs in III-V 
semiconductors that are Zincblende in bulk form, Glas, Harmand, and Patriarche argued correctly that nuclei in the VLS growth mechanism are energetically favorable to form at the triple-phase (vaporliquid-solid) boundary at the edge of the NW. ${ }^{54}$ Using environmental transmission electron microscopy, Gamalski et al. monitored the nucleation and growth of Ge NWs from a Au-Ge growth seed, and showed that nucleation is initiated at the edges of truncated NWs (Figure 5). 55 From the changes in the contact angle and corner area, they quantified a cyclic supersaturation mechanism which correlated the nucleus formation at the NW edge with an incubation time after which a ledge propagates and new (111) layer forms at the NW tip. Oh et al. made similar observations in $\alpha-\mathrm{Al}_{2} \mathrm{O}_{3}$ NWs grown from $\mathrm{Al}$ growth seeds (Figure 5f). ${ }^{56}$ Wen et al. recorded this behavior in $\mathrm{Si}, \mathrm{Ge}$, and $\mathrm{GaP}$ NWs (Figure $5 \mathrm{~g}-\mathrm{h}$ ). ${ }^{57}$ They also quantified the change in the area of the truncated liquid $\mathrm{Au}-\mathrm{Si}$ at a $\mathrm{Si}$ NW edge below the growth-seed/Si flat interface (Figure 5i) showing the periodic morphology change. These relatively new insights into the NW nucleation have significant implications for dopant incorporation in NWs as we will discuss next.
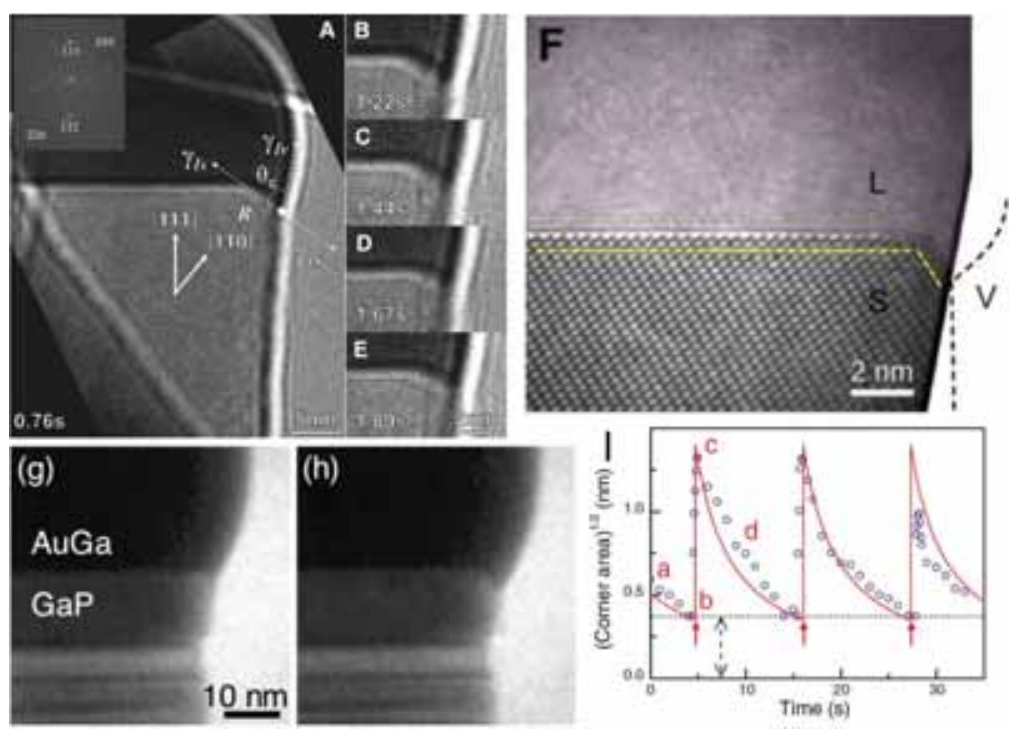

Figure 5: (a) - (e) In-situ TEM observation of periodic changes in the volume of the AuGe (dark) alloy area at the edge of a Ge NW (gray). Copyright American Chemical Society. Reproduced with permission from ref. [55]. (f) In-situ TEM captured image during the growth of $\alpha-\mathrm{Al}_{2} \mathrm{O}_{3} \mathrm{NWs}$ grown from Al growth seeds showing a truncated edge. Copyright Science Magazine. Reproduced with permission from ref. [56]. (g) - (h) In-situ TEM captured image during the growth of GaP NWs. (i) periodic change of corner area of the liquid AuSi alloy below the flat interface of AuSi and a [111] Si NW (not shown) Copyright American Physical Society. Reproduced with Permission from ref. [57]

Schwalbach, ${ }^{58}$ Perea, ${ }^{53}$ and Connel159 et al. developed an intuitive model that captures dopant incorporation in NWs during growth, which they quantitatively measured by local electrode atom probe (LEAP) microscopy. In their 1D model, they assume that the local equilibrium exists at the liquid-solid interface such that the chemical potentials do not change across the interface such that the growth rate is zero. The basic framework for the model is depicted for the case of a phosphorous 
doped-Ge NW as depicted in Figure 6a. This allows to equate liquid and solid compositions to their

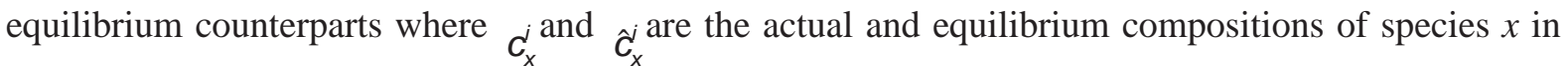
phase $i$. Assuming a phosphorous dopant flux $J_{p}$ that is smaller than the Ge flux $J_{G e}$, one can find the catalyst mediated doping composition to be

$$
C_{P}=\frac{\hat{C}_{P}^{\prime}}{\hat{C}_{P}^{s}} \cdot c_{P}^{s}=\frac{1}{k} \cdot \frac{J_{P}}{J_{P}+J_{G e}} \cong \frac{1}{k} \frac{J_{P}}{J_{G e}}
$$

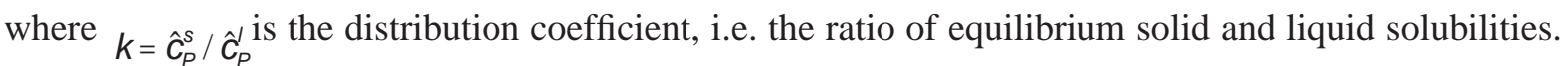

59 The doping efficiency is therefore limited by the flux ratios or dopant partial pressures, as is the case for bulk, and to the distribution coefficient of the liquid-solid phases.

At the liquid-solid interface, a mass balance equation can be written as:

$$
c_{P}^{l s}(k-1) \vec{v} \cdot \vec{n}=D \vec{\nabla} c_{P} \cdot \vec{n}
$$

where $c^{s i s}$ the composition of the liquid at the liquid-solid interface, $\vec{v}$ is the NW growth velocity, $\vec{n}$ is an outward pointing normal to the liquid-vapor interface..$^{59}$

At the liquid-vapor interface, another mass balance equation can be written,

$$
c_{P}^{l v} \vec{v} \cdot \vec{n}+D \vec{\nabla} c_{P} \cdot \vec{n}=J_{P} / \rho_{o}
$$

where $\rho_{o}$ is the density of the liquid. A supersaturation of Ge or undersaturation of $\mathrm{P}$ at the liquid-solid drives the growth. These small changes in composition from the local equilibrium can be used to determine the $\mathrm{P}$ composition in the solid and liquid from,

$$
\vec{v} \cdot \vec{n}=M R_{g} T(k-1) \int_{L} \frac{\left(c_{P}^{l s}-\bar{c}\right)}{L} d s
$$

where $M$ is the facet growth velocity normalized to supersaturation, termed as the facet mobility, $L$ is the length of the facet, $S$ is the arc length along the interface, and $\bar{c}$ is flux-dependent local equilibrium P concentration. Using equations (6) - (8), Connell et al. plotted the P concentration in a $\mathrm{Au}-\mathrm{Ge}$ droplet and found a local maxima above the truncated edges of the Ge NW as shown in Figure 6b. In their LEAP analysis, they found an enhanced P dopant concentration at the perimeter of the NW after turning off the $\mathrm{PH}_{3}$ source where the $\mathrm{P}$ dopants are believed to deposit from the Au-Ge droplet. Strikingly, the measurements pointed to maxima at the corner facets as illustrated Figure $\mathbf{6 d}$ and Figure 6e in agreement with their model described above. 


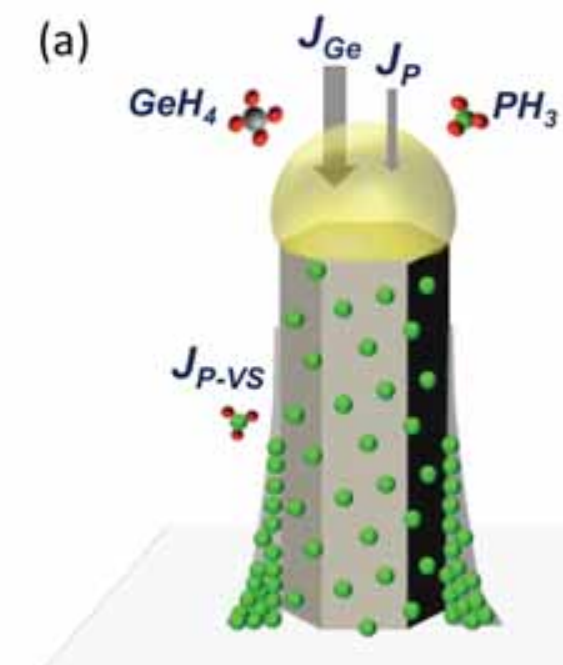

(b)

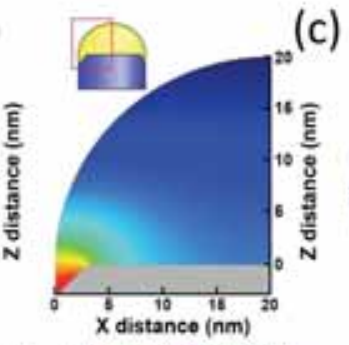

(c)

(d)
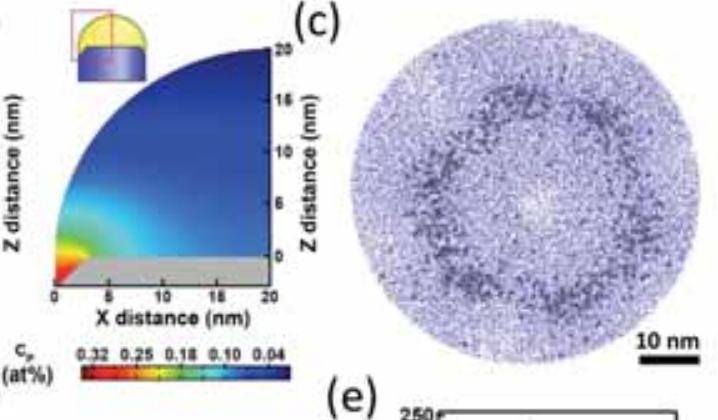

(e)
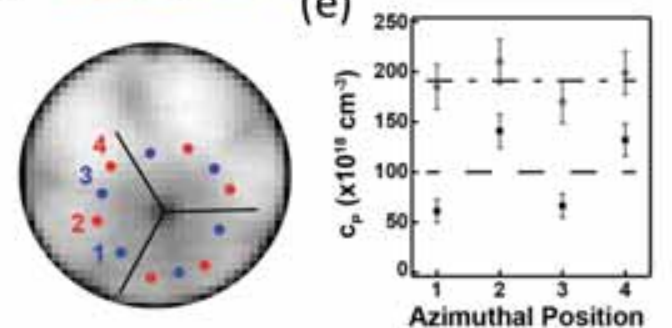

Ge (111)

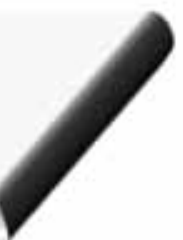

Figure 6: Doping mechanism and dopant gradients in radial direction: (a) Schematic illustration of phosphorous doping of a Ge NW with direct incorporation through the liquid growth seed or through the direct vapor-solid deposition at the sidewall. (b) Phosphorous dopant distribution in a liquid droplet with a truncated edge. (c) Measured dopant distribution in a VLS grown NW (directly after switching off $\mathrm{PH}_{3}$ source) buried in an undoped shell, i.e. incorporated from the liquid droplet. (d) Dopant concentrations overlaid on a Ge hit map density showing azimuthal anisotropy in doping as across the NW cross-section as plotted in (e) for numbered locations in (d). Panels (b) - (e) adopted with permission from ref. [59]. Copyright American Chemical Society.

The finite solubility of $\mathrm{PH}_{3}$ in the ternary Au-Ge-P liquid droplet has also detrimental effects on the axial doping profiles in what is commonly known as a reservoir effect. As the doping precursor is switched off, the finite doping concentration in the droplet will gradually reduce to negligible values, which can only be accomplished through the gradual deposition of the 'stored' dopants in NW segments that are not intended to be doped. This effect is present in systems that crystalize from liquids, has been known for decades in liquid phase epitaxy, ${ }^{60}$ and was recently discussed in the context of axial heterostructure NW growth. ${ }^{61,62,63}$ In their LEAP studies, Connell et al. also identified such doping gradients in a Ge NW as shown in Figure 7. The P concentration decays exponentially in the non-intentionally doped segment of the Ge NW according to

$c_{P}^{s}(z)=k \hat{c}_{p}^{\prime} \exp \left(-\frac{3 k\left(z-z_{0}\right)}{d}\right)$

where $z_{0}$ is the point at which the $\mathrm{PH}_{3}$ flow was terminated. It is possible that longer doping gradients would be measured if the sensitivity of the measurement technique is higher than that of LEAP or the number of other impurities (eg. Au or O) in the grown NW are low. 


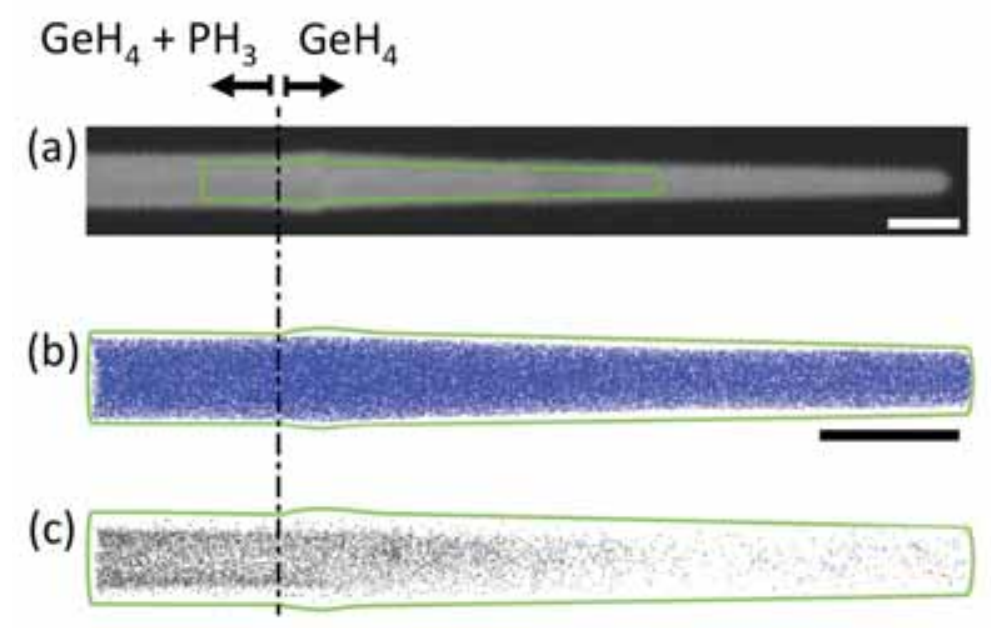

(d)

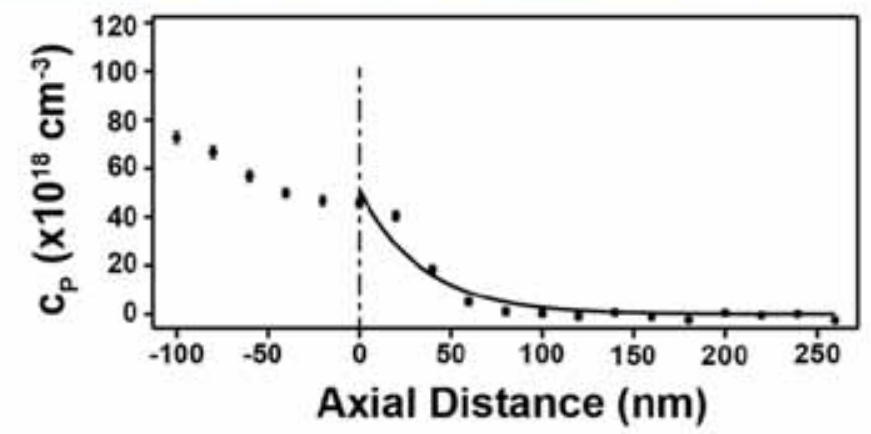

Figure 7: Dopant gradients in axial direction: (a) SEM image of a Ge NW grown with $\mathrm{PH}_{3}$ flow and after $\mathrm{PH}_{3}$ flow was terminated. (b) LEAP extracted Ge concentration. (c) LEAP extracted $\mathrm{P}$ concentration showing presence of $\mathrm{P}$ post termination of $\mathrm{PH}_{3}$ flow. (d) Linear profile of $\mathrm{P}$ concentration along the NW axis showing significant residual $\mathrm{P}$ dopant in Ge NW region grown without $\mathrm{PH}_{3}$ flow. Reproduced with permission from ref. [59]. Copyright American Chemical Society.

In addition to the axial dopant profiles, there is another competing doping mechanism in VLS grown NWs through the direct deposition of dopants on the NW sidewalls during growth. While the liquid catalyst mediates the anisotropic growth of 1D crystals through the VLS mechanism, nonidealities as sidewall deposition usually exist during NW growth. This is schematically illustrated in Figure 6a where dopant incorporation through sidewall deposition from direct crystallization from the vapor to a solid shell is termed as $J_{P \text {-VS }}$. The significance of this effect is directly related to the growth conditions, where higher temperatures and dopant partial pressures as well as uncontrolled precursor heterogeneous reactions are the dominant factors. Figure 8 illustrates these effects in a P-doped Ge NW grown under continuous flow of both $\mathrm{GeH}_{4}$ and $\mathrm{PH}_{3} .{ }^{53}$ The circumference-to-center doping concentration ratio can be as large as two orders of magnitude underlining another significant limitation of doping in VLS grown NWs which is uncontrolled sidewall deposition. The effect has also been verified with other measurement techniques, such as Kelvin Probe Force Microscopy (KPFM) on the same type of NWs, ${ }^{64}$ and spreading resistance measurements on Si NWs. ${ }^{65}$ At a diameter of few nanometers, there are other size-related thermodynamic considerations that favor dopants to segregate to NW surfaces. ${ }^{66,67}$ Xie et al. ${ }^{68}$ investigated the spatial distribution of dopants in 
Si and Ge NWs, and found a "transition diameter" about 22 25nm, below which the body doping was low and most of the dopants can be located at the surface layer. Overall, these dopant gradient profiles can hinder the reach of NWs to some important applications that require the formation of an abrupt junction and steep dopant profiles.

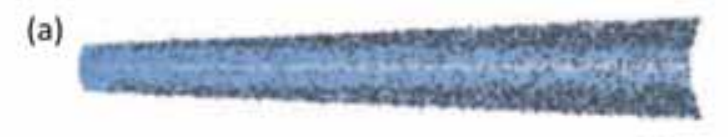

(b)
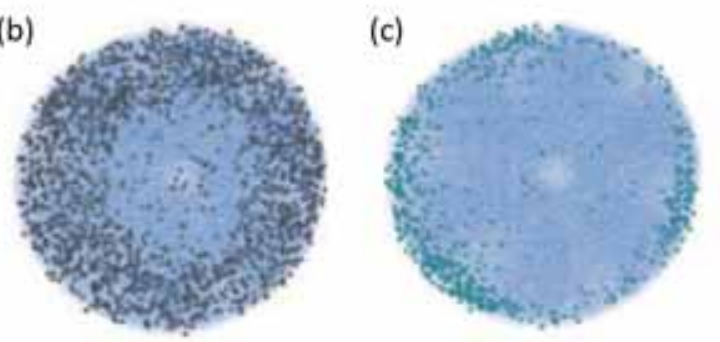

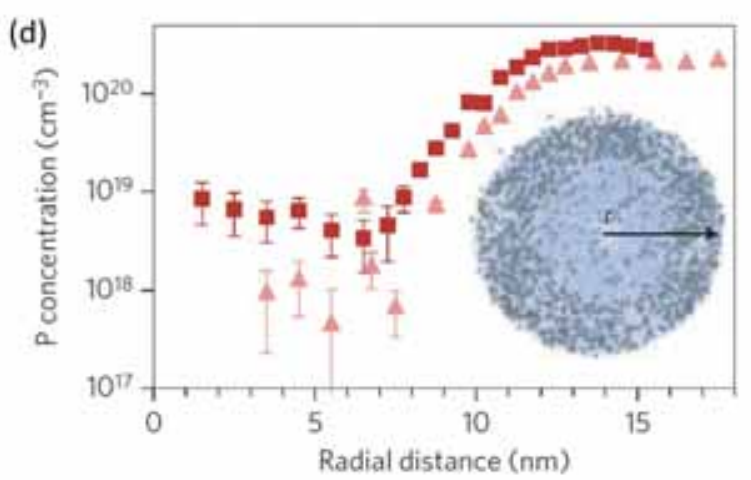

Figure 8: Radial doping gradients in a P-doped Ge NW. (a) 2nm thick slice of a P-doped Ge NW. (b) Cross-section showing spatial distribution of $\mathrm{P}$ atoms (black), and $\mathrm{O}$ atoms (green) in (c). (d) Extracted radial $\mathrm{P}$-concentration showing significant sidewall doping through $\mathrm{J}_{\mathrm{P}-\mathrm{vs}}$ and lower $\mathrm{P}$ dopant concentration in the center of the NW. Squares and circles are for a $\mathrm{PH}_{3}: \mathrm{GeH}_{4}$ ratios of 1:1000 and 1:100, respectively. Reproduced with permission from ref. [53]. Copyright of American Chemical Society.

\section{Influence of Doping on Elemental Nanowire Growth Morphology}

Introduction of dopants during NW growth can significantly alter their morphologies. As early as 1970s, Givargizov et al. ${ }^{69,70}$ investigated the role of $\mathrm{AsCl}_{3}$ doping on $\mathrm{Si}$ and Ge whisker growths. They found that the Si whiskers had periodic instability of diameters during growth at certain growth conditions, and that Ge whiskers tended to have a larger catalyst head named as "fungaceous whisker". A small amount of $\mathrm{AsCl}_{3}$ doping (1\% to $\mathrm{SiCl}_{4}$ or $\mathrm{GeCl}_{4}$ ) could significantly suppress the oscillations in Si whisker growth and change the "fungaceous whisker" to a regular wire shape. The role of $\mathrm{AsCl}_{3}$ here, as they pointed out, was to reduce the surface tension at the liquid-solid interface, changing the shape of liquid catalyst at the NW tip (schematically illustrated in Figure 9a) and suppress the self-oscillating effect of Au droplet. We observed such instabilities in 2006 during the growth of In-catalyzed InAs NWs ${ }^{37}$ and this phenomenon was utilized for crystal engineering in III-V materials where alterations between zincblende and wurtzite segments was precisely controlled. ${ }^{18}$ Theoretically, introducing dopant in such NW growth conditions may either promote or annihilate instabilities of NW morphology for different application purposes. Schwarz and Tersoff have 
eloquently employed the dynamic changes at the growth interface to explain the various NW morphologies. ${ }^{71}$ Such oscillations were used to interpret kinking in a variety of NW systems. ${ }^{72,73,74}$

$\mathrm{PH}_{3}$ and $\mathrm{B}_{2} \mathrm{H}_{6}$ are most widely used as doping precursors for n-type and p-type Si/Ge NWs. $\mathrm{PH}_{3}$ doping is generally found to have negligible effect on $\mathrm{NW}$ morphologies, ${ }^{75,76}$ while $\mathrm{B}_{2} \mathrm{H}_{6}$ is found to affect the NW morphologies in many different ways. ${ }^{14,77-79}$ Lew et al. ${ }^{77}$ reported that B-doped $\mathrm{Si}$ NWs have crystalline cores and amorphous shell as schematically illustrated in Figure 9b. Tutuc et al. 78 found a large sidewall overgrowth of B-doped Ge NWs, resulting in a tapered NW shape (schematics in Figure 9c). This is attributed to the low decomposition temperature of $\mathrm{B}_{2} \mathrm{H}_{6}$ and the facility of homogenous gas reactions between $\mathrm{B}_{2} \mathrm{H}_{6}$ and $\mathrm{NW}$ source gases, as has been shown with $\mathrm{SiH}_{4}$ resulting in increased $\mathrm{Si}$ growth rate on the NW sidewalls. ${ }^{80,81}$ The strong tapering during growth leads to accumulation of thicker doped shells near the base than near the tip leading to strong dopant gradients between base and tip. ${ }^{82} \mathrm{Li}$ et al. ${ }^{79}$ studied the axial grown p-n-i Si NWs, and found that only the p-type region showed saw-tooth like surface facets (Figure 9d and Figure 10). Faceting in NW growth can be introduced by commonly known twining 83 or self-oscillations in liquid catalyst, ${ }^{84}$ which usually happens at low precursor partial pressures. In the absence of these two causes, Li et al. attributed the sawtooth morphology to $\mathrm{B}_{2} \mathrm{H}_{6}$ enhanced VS growth on the NW sidewalls. Uesawa et al. later supported the same behavior in $\mathrm{Si} \mathrm{NW}$ growth through the $\mathrm{Zn}$ reduction of $\mathrm{SiCl}_{4}{ }^{85} \mathrm{Xu}$ et al. have shown conclusively that under low $\mathrm{SiH}_{4}$ partial pressures, Au clusters on the microwire surface induce surface reconstruction of the $\{112\}$ facets which have a sawtooth like morphology, ${ }^{86}$ as will be further elaborated in section 1.5 .

(a)

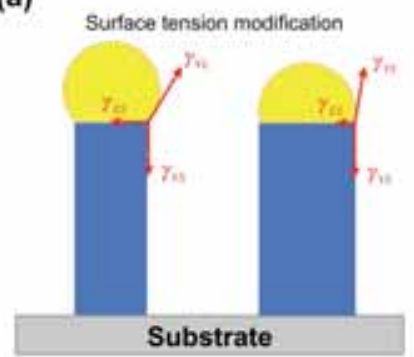

(c)

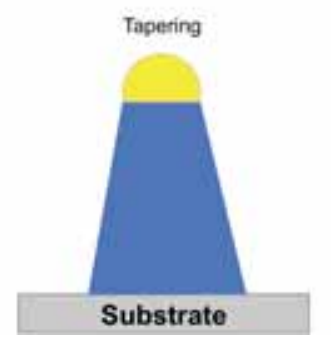

(b)

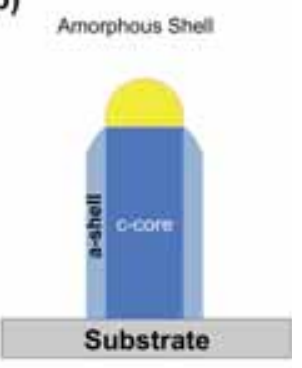

(d)

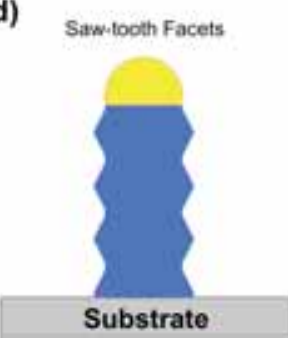

Figure 9: Schematics showing the doping effects on NW morphologies. 

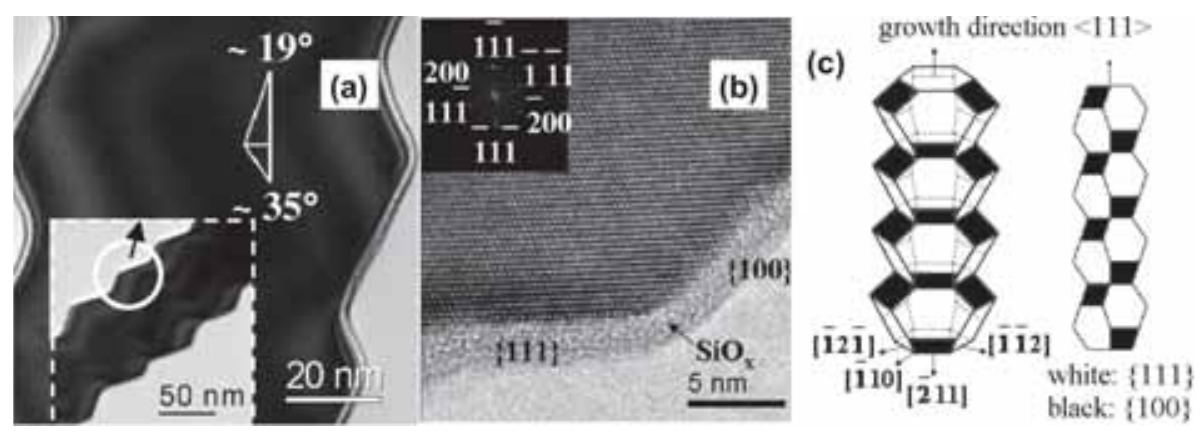

Figure 10: $\mathrm{B}_{2} \mathrm{H}_{6}$ doped $\mathrm{Si} \mathrm{NW}$ with saw-tooth shaped surface facets. (a) and (b) TEM images of $\mathrm{Si}$ NW sidewall facets. (c) Schematics of the sidewall facets, in which $\{111\}$ and $\{100\}$ are the dominant facets. Reprinted with permission from the American Institute of Physics, copyright 2009, ref. [79].

To suppress the $\mathrm{B}_{2} \mathrm{H}_{6}$ induced $\mathrm{Si}$ deposition on NW sidewalls, one approach is to increase the hydrogen partial pressure which can reduce the Si deposition rate. ${ }^{87,88}$ Another approach is to replace $\mathrm{B}_{2} \mathrm{H}_{6}$ with trimethylborane (TMB), as TMB has a higher decomposition temperature and was found to introduce negligible morphology changes during NW growth even at very high dopant/semiconductor ratios. 76,77

Another approach to uniformly dope NWs along the NW axis with smooth surface morphology is to grow undoped NW cores and elevate the temperature to catalyze the vapor-solid crystallization of doped shells. For the case of P-doped Ge shells, we have shown that this approach leads to crystalline and uniformly doped shells in a 1:7500 $\mathrm{PH}_{3}: \mathrm{GeH}_{4}$ ratio (Figure 11a). Two-terminal devices made on these NWs showed negligible resistivity $\left(\varrho_{s}=10^{-3} \Omega . c m\right)$ changes across the entire NW as shown in Figure 11b-c. When the same method was applied for B-doped Ge NWs (1:7500 $\mathrm{B}_{2} \mathrm{H}_{3}: \mathrm{GeH}_{4}$ ), we attained smooth shells but with some stacking faults despite the B-doped shells were only $3 \mathrm{~nm}$ thick. Additional studies are required to rule out the cause of defect evolution in B-doped shells. Overall, this approach seems reasonable to attain uniform axial doped NWs for applications that might not be sensitive to abrupt radial changes in dopant concentration such as in radial photodetectors or solar cells. 

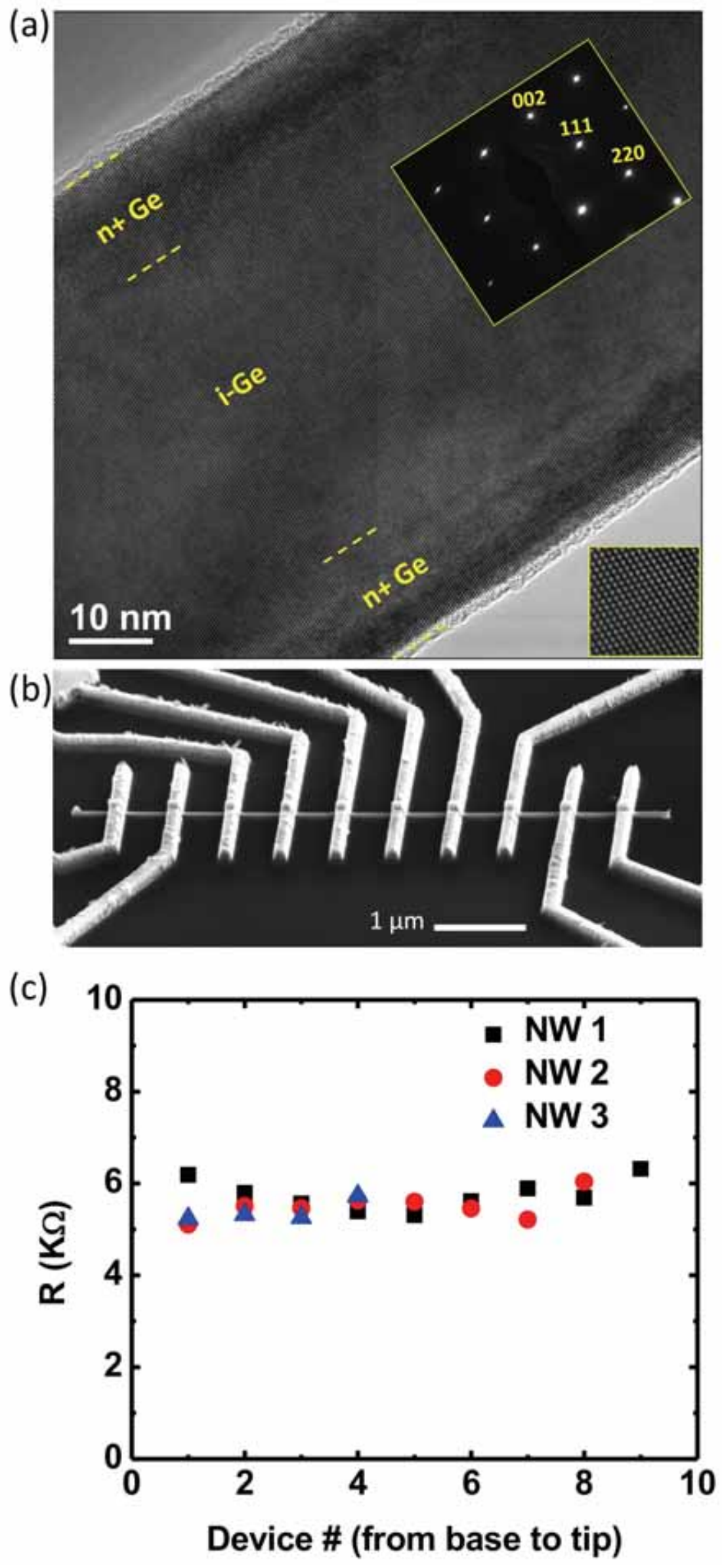
Figure 11: (a) TEM image of a 40nm diameter Ge NW coated with a 13nm uniformly P-doped shell. (b) Test device on a Ge NW from the same growth run as that in (a) with $130 \mathrm{~nm}$ wide Co contact metal (NW1, NW2). (c) The resistance of $3 \mathrm{NW}$ devices taken from base to tip showing uniformity in doping. NW3 device had 560nm wide Co contacts. Separate transmission line measurements (variable separation between contacts to extrapolate to zero separation for contact resistance extraction) showed a contact resistivity of $10^{-6} \Omega . \mathrm{cm}^{2}$.

\section{Influence of Doping on Compound Semiconductor Nanowire Morphology}

Growth of compound semiconductor NWs is generally more complicated than elemental semiconductor NWs, as more than one reactant precursor is required in the VLS process. Many competitive processes, such as radial and substrate growths and substrate-NW adatom exchange are more prominent than the case of $\mathrm{Si}$ and Ge NWs. ${ }^{89}$ For each species in the compound semiconductors NWs, its precursor has different decomposition rates in the vapor phase, diffusion lengths on the substrate and NW sidewall surfaces, and sticking efficiencies at the growth seed particles. Several preceding review papers have elaborately introduced the VLS growth mechanisms for compound semiconductors. ${ }^{90-92,93,94}$ The morphology of undoped NWs can be tuned with the growth parameters. 37,89 When doping and crystal phase requirements restrict the growth windows, other techniques that can result in optimal morphology are desired. Borgström et al. utilized in-situ $\mathrm{HCl}$ flow to eliminate deposition on the sidewalls of InP NWs at growth conditions that otherwise favor tapering (Figure 12). ${ }^{95}$ This facilitated the growth of well-behaving InP axial NW solar cells ${ }^{96}$ and the elimination of InP deposition on the Si substrate in axial InP NW tandem junction cells. ${ }^{97}$ Wallentin et al. found that $\mathrm{H}_{2} \mathrm{~S}$ flow during growth could reduce the NW radial growth and increase the axial growth by more than three times due to the passivation effect on the NW sidewall. ${ }^{8}$ If the NW was grown in the presence of $\mathrm{HCl}$, by which the radial growth had been already eliminated, the $\mathrm{H}_{2} \mathrm{~S}$ wouldn't noticeably change the growth rate. ${ }^{99}$
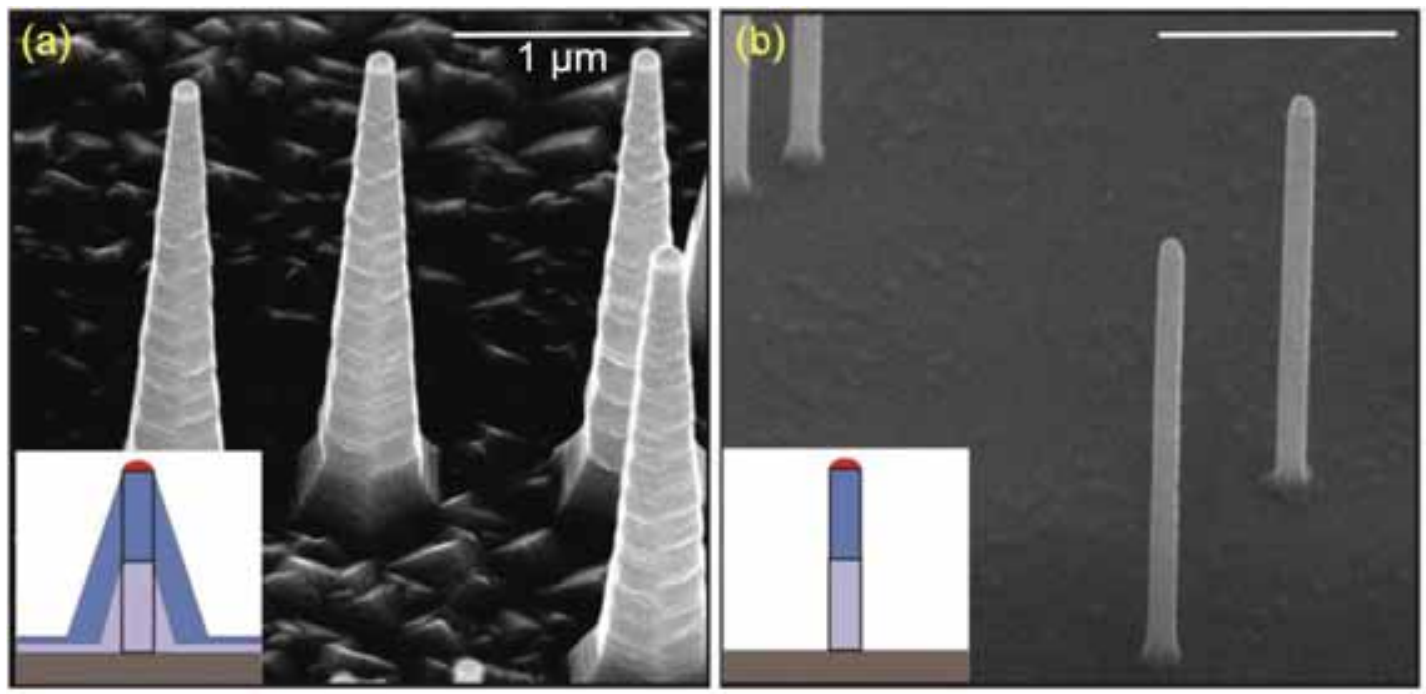

Figure 12: SEM images of undoped InP NWs grown (a) without and (b) with $\mathrm{HCl}$ flow at a molar fraction of $2.9 \times 10^{-5}$ under otherwise similar growth conditions. Insets cartoons are hypothetical 
schematics of axial junctions that may be compromised if the $\mathrm{HCl}$ is not flown during growth. Reproduced with permission from ref. [95].Copyright Tsinghua University Press and Springer-Verlag Berlin Heidelberg 2010.

It was argued that doping effects on the growth rate and crystalline structures are often stronger than those by changing growth parameters, such as V/III ratio. ${ }^{100}$ First, incorporation of dopants into compound NWs depends on both the solubility of dopants in liquid catalyst and their segregation coefficient $\left(k_{L S}=C_{S} / C_{L}\right)$ at the liquid-solid interface. Wallentin et al. ${ }^{100}$ summarized the dopant incorporation in two extreme situations: type A, in which dopant has low solubility but a large $k_{L S}$. Therefore incoming dopant flux into the liquid droplet $\left(J_{D}^{V L}\right)$ equals to the flux incorporated into the solid NW ( $J_{D}^{L S}$ ), leading to a kinetic controlled process; type B, in which the dopant has high solubility but a small $k_{L S}$ and therefore the doping concentration is determined by $C_{S}=k_{L S} \cdot C_{L}$, leading to a thermodynamic controlled growth regime. A good example of type A dopant is sulfur (S) in InP, ${ }^{101}$ and the counterpart example of type B dopant is $\mathrm{Si}$ in Ga-catalyzed GaAs. ${ }^{102}$ Second, the relative fluxes or partial pressures of anions and cations will also affect the incorporation of dopants. 103 During the metalorganic CVD (MOCVD) process for III-V NWs, the vapor phase precursors are trimethylindium (TMln), trimethylgallium (TMGa) for group-III constituents, and $\mathrm{AsH}_{3}, \mathrm{PH}_{3}$ typically for group-V constituents. Group II elements, such as $\mathrm{Zn}$, can be used as p-dopants to occupy group-III lattice sites, and group-VI elements, such as S, can be used as n-type dopants to occupy group-V lattice sites. Therefore, a relatively low V/III ratio can facilitate incorporation of n-type dopant, while a higher V/III ratio will enhance p-type doping. Generally, group-V elements have much lower solubility in liquid metallic droplet than group-III elements, leading to difficulties in achieving highly p-type doped NWs. ${ }^{104}$ Finally, the largest difference from elemental semiconductor NW is that there exist amphoteric dopants (such as $\mathrm{Si} / \mathrm{Ge}$ for III-V compound NWs) that can serve as both p-type and n-type dopant depending on their occupation sites in the NW crystal. ${ }^{105,106}$ The lattice locations of amphoteric dopants are determined by many parameters, such as compound stoichiometry, Fermi level, and solubility limit, and can be adjusted by tuning the NW growth condition. ${ }^{107}$ It has been reported that with only Si doping species, both a p-type core and an n-type shell in GaAs p-i-n structure were grown by controlling the distinct growth conditions for the core and the shell. ${ }^{108}$

One the other hand, the dopant incorporation can also affect growth of compound semiconductors. Dimethylzinc (DMZn) was found to increase the NW growth rate, 109 while 
diethylzinc (DEZn) does not. ${ }^{110}$ This is due to the higher decomposition temperature of DMZn that allows vapor phase DMZn to passivate the NW sidewall and substrate surface and enhance the axial growth rate. However, doping at very high molar ratios may completely terminate the NW growth, 110,111 or introduce kinks in the NW structure. ${ }^{98,99} \mathrm{Si}$ doping in InP NW was found to increase the tapering and reduce the axial growth rate. ${ }^{112}$ Most interestingly, doping may also modify the compositions for ternary semiconductor NW. Wallentin et al. ${ }^{113}$ reported the growth of $\operatorname{Ga}_{x} \operatorname{In}_{(1-x)} P$ NWs with $p-i-n$ radial junction. They found that the $S$ doped region is slightly more In rich $(x=0.7)$ than the undoped region ( $x=0.77$ ), due to the $\mathrm{H}_{2} \mathrm{~S}$ modified sidewall facets that affects the diffusion length of groups III and V adatoms. In DEZn doped region, there's a gradient of Ga content from $\mathrm{x}=0.63$ (doping start) to $\mathrm{x}=0.85$ (doping stabilize) due to the $\mathrm{Zn}$ affected equilibrium concentration of $\mathrm{Ga}$ in the liquid droplet. Moreover, the doping compensation effect should also be considered when using amphoteric dopants during compound semiconductor NW growth. As will be further discussed in section X.102

\section{Side Effects During Nanowire Growth: Impurity Contamination from Au Growth Seeds}

The previous sections discussed the VLS mechanism and modified growth processes with dopant introduction for both elemental and compound semiconductor systems. One more aspect during the NW VLS growth that often draws underrated attention is the incorporation of metallic catalyst during NW growth. Even though the growth seed impurity may serve as dopant during NW growth in some cases, such as Al-seeded Si NW growth, ${ }^{114,115}$ in most other cases the incorporation of growth-seed impurities are considered notorious. Au is used as the predominant catalyst in almost all the NW growths discussed above, but Au contamination has long been known detrimental to semiconductor device performances. Au forms mid-gap traps in elemental semiconductors which act as recombination centers for excess electron and hole pairs, and significantly decrease the minority carrier lifetimes. ${ }^{116,117}$ Therefore, it's essential to understand the distribution and quantity of $\mathrm{Au}$ impurities in VLS grown NWs, and their impact on device performances.

Au contaminants can segregate in three different locations during NW growth: NW body, defect sites, and NW sidewall. Perea et al. were the first to quantitatively determine the NW composition by LEAP microscopy (Figure 13) that Au incorporates into the grown InAs NW body by the VLS growth at a concentration of 100 atomic parts per million. ${ }^{118}$ This corresponds to a very high concentration of $\sim 3.5 \times 10^{18} \mathrm{~cm}^{-3}$. Naturally, this concentration is heavily dependent on the growth conditions; the examined NWs in Perea et al.'s report seem to be non-tapered and therefore might have been grown near optimal conditions. For Ge NWs analyzed by the same group using LEAP, it was found that the Au concentrations in both undoped and phosphorous doped Ge NWs are $<2 \times 10^{17}$ $\mathrm{cm}^{-3}$, and oxygen concentration $<3 \times 10^{17} \mathrm{~cm}^{-3}$ for undoped Ge NWs and $3-12 \times 10^{17} \mathrm{~cm}^{-3}$ for P-doped 
Ge NWs. ${ }^{119}$ Through the analysis of measured conductivities these Ge NWs, they found that surface states overwhelmingly dominate the doping and free carrier concentration in Ge NWs, which is reasonable given the well-known surface Fermi energy pinning at or the near the charge neutrality level in $\mathrm{Ge}$ at about $~ 90 \mathrm{meV}$ above the valence band-edge. ${ }^{120}$

Secondary ion mass spectrometry (SIMS) is a standard technique in the compositional and dopant mapping in semiconductors. ${ }^{121}$ Using SIMS, Putnam et al. have shown that the upper limit of $\mathrm{Au}$ in VLS grown Si microwires (MWs) using $\mathrm{SiCl}_{4}$ at $1000{ }^{\circ} \mathrm{C}$ was $1.7 \times 10^{16} \mathrm{~cm}^{-3}$ (Figure 13 (d) (e)), in agreement with the bulk thermodynamic equilibrium concentration at $1000^{\circ} \mathrm{C}\left(10^{16} \mathrm{~cm}^{-3}\right) .122$ They calculated a $3 \mathrm{~ns}$ minority carrier lifetime for both electrons and holes at a doping density of $10^{18}$ $\mathrm{cm}^{-3}$ leading to $\sim 1 \mu \mathrm{m}$ diffusion lengths which is in agreement with their scanning photocurrent microscopy measurements and diffusion length estimates. ${ }^{123}$ The same group later resorted to using Copper as a VLS growth seed, which can segregate to the MW surface post-growth and can be fully chemically etched as validated by surface-sensitive x-ray photoelectron spectroscopy. ${ }^{124}$
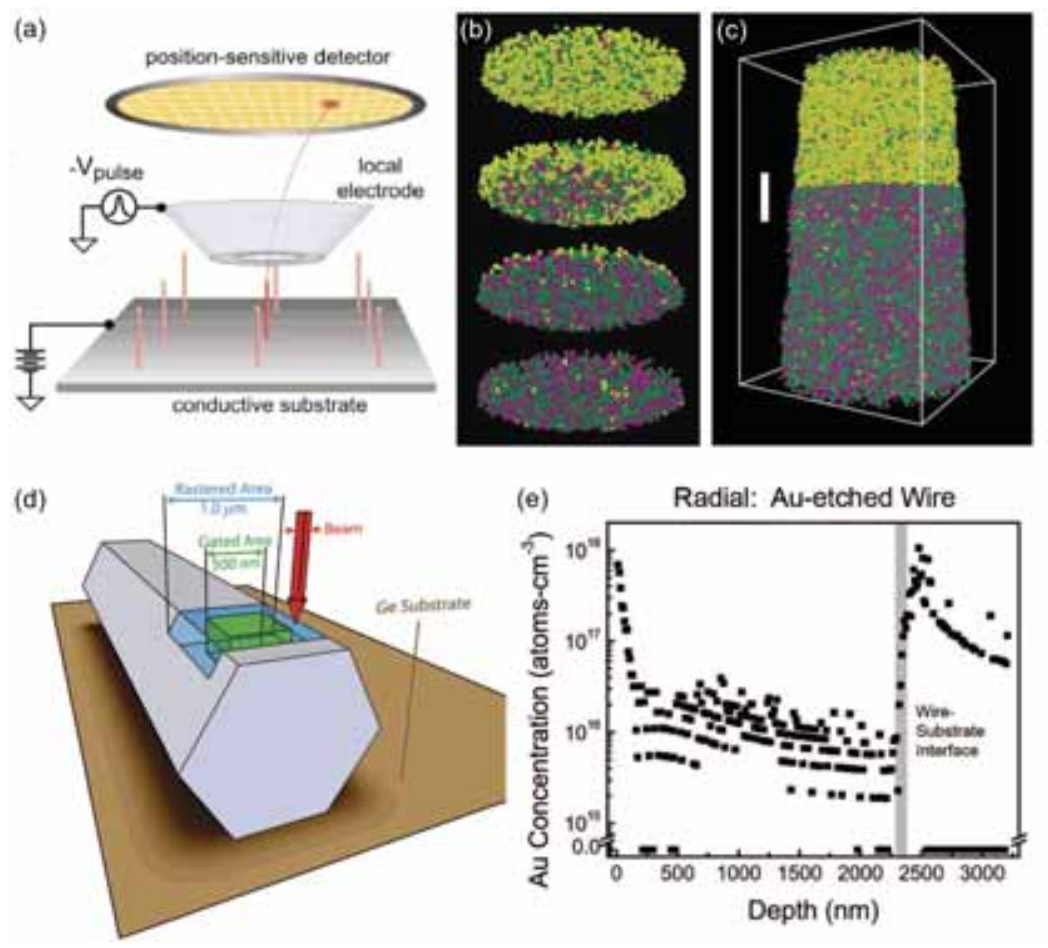

Figure 13: Analysis of Au composition in VLS InAs (a) - (c) and Si (d) - (e) NWs. (a) Schematic illustration of the LEAP composition microscopy in NWs. (b) Three dimensional reconstruction of a Au-InAs NW interface using LEAP with $1 \mathrm{~nm}$ thick slices at the Au-InAs interface. (c) Reconstruction of the InAs NW tip in a $14 \times 14 \times 23 \mathrm{~nm}^{3}$. Reprinted with permission from the American Chemical Society, copyright 2006, Ref. [118]. (d) Schematic illustration of SIMS analysis of Au composition in VLS Si NWs. (e) Radial Au concentration profile in a Si NW. Reprinted with permission from the American Chemical Society, copyright 2008, Ref. [122].

Using high angle annular dark-filed (HAADF) imaging under aberration-corrected scanning transmission electron microscope (STEM) mode, Allen et al. determined the location of Au atoms in Si NWs. ${ }^{125}$ They found that Au atoms incorporated in NW body (marked '1' in Figure 14a), with 
some Au atoms located at NW surface (marked '2' in Figure 14b). They also observed aggregated Au atoms at the twin interface of a defect NW (shown in Figure 14b). Incorporation of those Au atoms into NW body was attributed to the non-equilibrium process in supersaturation of catalyst droplets during NW growth, rather than diffusion of $\mathrm{Au}$ after the growth process as supported by LEAP analysis. By analyzing the complete mass spectra, they provided an estimation of the upper bound of incorporated $\mathrm{Au}$ atoms $\sim 5 \times 10^{17}-1.5 \times 10^{18}$ atoms $/ \mathrm{cm}^{3}$ in the NW body, which is much higher than the thermal equilibrium solubility of $\mathrm{Au}$ in $\mathrm{Si}$. The incorporated $\mathrm{Au}$ atom concentration was estimated to have a similar value $\left(10^{17} \sim 10^{18}\right.$ atoms $\left./ \mathrm{cm}^{3}\right)$ in Au-catalyzed GaAs NWs, counted from HAADF images with a series of focal depths. ${ }^{126}$
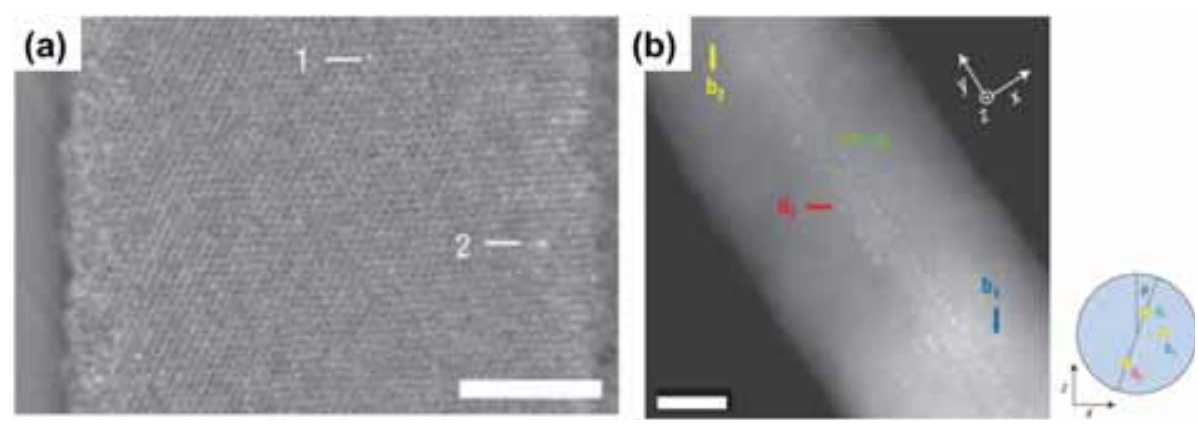

Figure 14: (a) Filtered HAADF-STEM image showing Au atoms in Si NW. (b) Sum of several aligned HAADF-STEM images of different focal depth, showing the aggregation of Au atoms near a twin interface. Scales bar are $5 \mathrm{~nm}$. Reprinted with permission from the Nature publishing group, copyright 2008 , Ref. [125]

Another contamination aspect is the Au wetting of Si NW sidewalls during growth. This was first introduced by in-situ observations of Si NW grown in ultra-high vacuum transmission electron microscope (UHVTEM) chamber. ${ }^{127}$ It was observed that liquid droplets changed size during the growth, enabled by the Au diffusion from the smaller droplets to the bigger ones in what's known as Ostwald ripening as shown in Figure 15a. Since $\mathrm{Au}$ has limited solubility in bulk $\mathrm{Si}$ (below $10^{13}$ atoms $/ \mathrm{cm}^{3}$ at the growth temperature $600{ }^{\circ} \mathrm{C} 128$ the authors attribute such significant Au migration to the wetting and diffusion of Au through NW sidewall and substrate surface instead of the NW body. From this hypothesis, they predicted that Au would be gradually consumed by wetting the NW sidewall and eventually terminate the NW growth. With longer growth times, it was proven that all the NWs had much smaller catalyst than the starting sizes of Au seeds (shown in Figure 15b). Den Hertog and Kawahima found that Si NWs grown with very low silane partial pressure and high temperature had tapered shapes and diffusion of Au towards NW sidewalls. ${ }^{129,130}$ Those Au clusters on NW sidewall can further modify the surface free energy and induce the structure reconstructions as shown in Figure 15c. ${ }^{86}$ Through detailed scanning tunneling electron microscopy, Xu et al. have shown that such sawtooth like structures in $\{112\}$ facets are generally a combination of $\{111\}$ and 
higher index facets of the families $\{113\},\{115\},\{117\}$ and $\{119\}$ where Au cluster adsorbates were found on the high index facets. ${ }^{86}$ Kodambaka et al. introduced $\mathrm{O}_{2}$ during growth to suppresses $\mathrm{Au}$ contaminations. ${ }^{131}$ Dayeh et al. have shown that such $\mathrm{Au}$ diffusion is diameter dependent and kinetically driven (Figure 16). ${ }^{132}$ By lifting up the surface energy density of a Ge NW sidewall using a thin Si rich SiGe segment underneath the Au growth seed, Au diffusion on the NW sidewalls was inhibited which enabled the growth of smooth Si shells on Ge NW cores. ${ }^{133}$

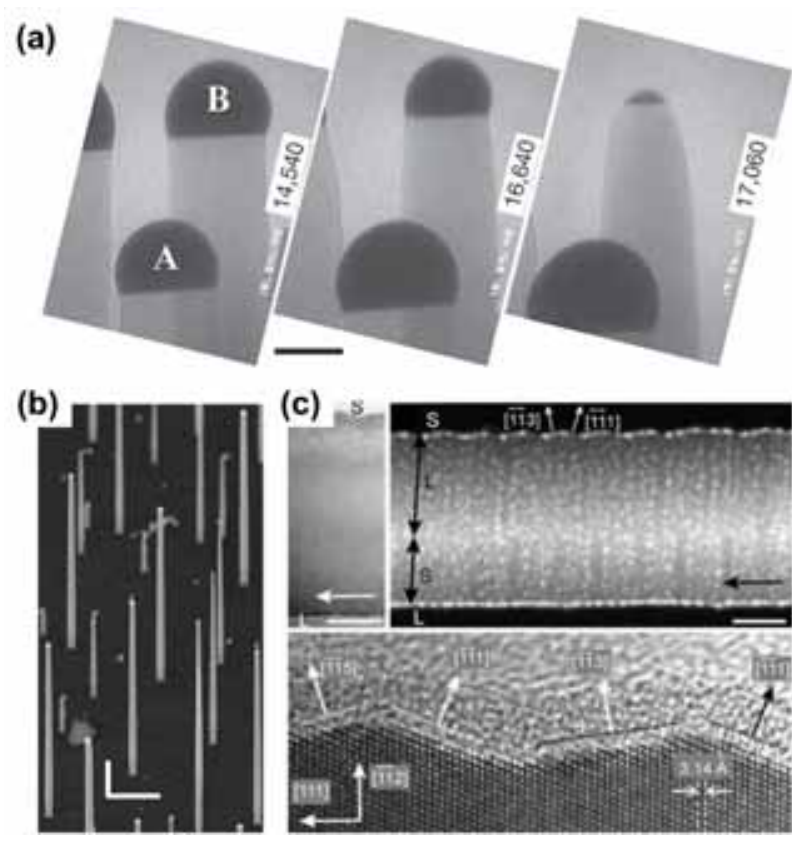

Figure 15: (a) In situ UHVTEM images at different time frames recorded during the Si NW growth. Scale bar is 50nm. (b) Si NWs grown for $1 \mathrm{~h}$ at $600{ }^{\circ} \mathrm{C}$ in a $20 \%$ disilane and $80 \%$ He solution at a pressure of $5 \times 10^{4}$ Torr. Scale bar is $1 \mu \mathrm{m}$. Reprinted with permission from the Nature publishing group, copyright 2006, Ref. [127] (c) HAADF and HRTEM images showing the Au clusters on the Si NW sidewall and the reconstructed surface structure. Scale bars are $20 \mathrm{~nm}$. Reprinted with permission from the American Physical Society, copyright 2010, Ref. [86]. 


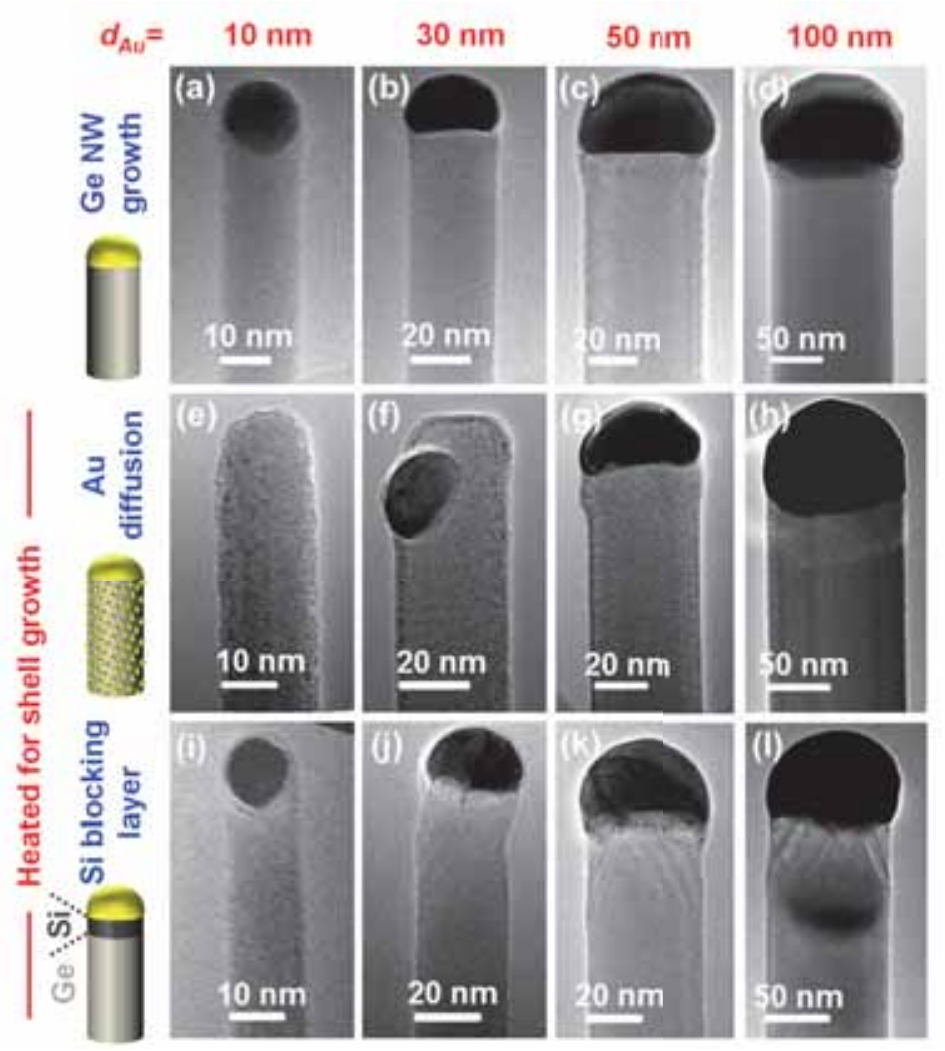

Figure 16: Elimination of the Au diffusion on Ge NW sidewalls by tailoring the NW surface energy. (a) - (d) As grown different diameter Ge NWs elongated at $276^{\circ} \mathrm{C}$ showing no Au diffusion. (e) - (h) NWs subject to a post-growth temperature ramp up to $410^{\circ} \mathrm{C}$ showing significant Au diffusion and loss of the Au growth seed at small diameters. (i) - (k) Au is stabilized at the NW tips through the introduction of $\mathrm{SiH}_{4}$ post growth at $276^{\circ} \mathrm{C}$ and the formation of a Si rich SiGe interfacial layer under the $\mathrm{Au}$ growth seed when subject to the same temperature ramp as in (e) - (h). Reprinted with permission from the American Physical Society, copyright 2010, Ref. [132]. This approach was used to growth the uniformly axial doped shells in Figure 11.

Such large concentrations of Au contamination in semiconductor NWs are detrimental to electronic devices that operate with minority carriers. Breuer et al. compared the optoelectronic properties of Au-catalyzed and self-assisted GaAs NWs core with the same AlGaAs shell. ${ }^{134}$ They found that the Au-catalyzed GaAs NW gave 2 orders of magnitude shorter minority carrier lifetime than self-assisted grown GaAs NWs, and subsequently a much lower internal quantum efficiency. Jiang et al. found out the proper AlGaAs surface passivation can also result in long minority carrier lifetimes of a few nanoseconds. ${ }^{135,136}$ On the other hand, Allen et al. ${ }^{125}$ found that the Au effect on minority carrier lifetime was diameter dependent. If the NW diameter was $\sim 100 \mathrm{~nm}$, the minority carrier lifetime is reduced due to recombination in Au trap states, while as diameter decreased, the minority carrier lifetime decreases due to recombination in surface trap states. While non-Au metal growth seeds have been utilized for semiconductor NW growth ${ }^{137,138}$, the ease of controlled diameter Au particle synthesis made them most widely used in NW growth and most researched in depth. 


\section{Measuring Dopant Concentration Profiles}

In addition to the LEAP and SIMS techniques discussed above, there are many methods to extract dopant or free-carrier concentration. The average dopant concentration in NWs can be measured from FET characteristics near flat-band voltages. ${ }^{139,140,141,142}$ Accurate measure of the gate capacitance $^{143}$ and free-carrier mobility ${ }^{144}$ and accounting for parasitic resistances and capacitances ${ }^{145,146}$ are required to obtain representative values for the doping concentration. Others utilized Hall, ${ }^{147,148,149}$ photocurrent, ${ }^{150}$, scanning photocurrent, ${ }^{151}$ thermoelectric ${ }^{152}$, and Raman ${ }^{153}$ measurements to extract the average NW carrier/dopant concentration. We believe that in addition to LEAP and SIMS, capacitance-voltage $(\mathrm{C}-\mathrm{V})$ provides the most accurate quantitative measure of dopant concentration and profile in grown NWs.

$\mathrm{C}-\mathrm{V}$ spectroscopy is conventionally a powerful tool to extract dopant and trap concentrations. In the first known vertical p-n junction devices on NWs, Haraguchi et al. utilized the slope of $1 / C^{2}(V)$ to extract the background dopant densities. ${ }^{9,154}$ Accurate knowledge of the dopant concentration requires performing CV characterization on individual NWs. In devices fabricated in the form of a field-effect transistor (FET), the source and drain contacts are usually shorted together, and to attain aF $\left(10^{-18} \mathrm{~F}\right)$ capacitance resolution, a capacitance bridge is used to cancel all electrode stray capacitances. ${ }^{155}$ Stray capacitance from probes needles can also be cancelled by the insertion of a grounded plate between the gate and the reference (source/drain) terminals as shown in Figure 17a.156 Garnett et al. utilized the capacitance bridge cancelation technique on a post-growth Boron doped Si NW by gas phase diffusion of $\mathrm{BCl}_{3}\left(d=75 \mathrm{~nm}\right.$, and $\left.\mathrm{t}_{\mathrm{Al2} 23}=15 \mathrm{~nm}\right) .{ }^{157}$ They applied a back-gate voltage $V_{b g}=-20 \mathrm{~V}$ to populate an accumulation layer of holes under the contacts to reduce their parasitic resistance during the NW C-V characterization, and the background capacitance was measured in the off-state at a $V_{b g}=8 \mathrm{~V}$ (Figure 17b). To properly extract the dopant profile, one must first eliminate the influence of interface trap and fixed oxide trap charges. Since high-frequency (HF) AC signals cannot modulate the interface trap charge character, the interface state capacitance will not appear in the HF small signal equivalent capacitance model. It must be included in the low-frequency (LF) capacitance model. The interface trap density can then be extracted from the two C-V curves (high-low method) using 157

$$
D_{i t}=\frac{\left(C_{L F}-C_{H F}\right)}{q\left(1-C_{L F} / C_{o x}\right)\left(1-C_{H F} / C_{o x}\right)}\left(\mathrm{cm}^{-2} \mathrm{eV}^{-1}\right)
$$

Figure 17c shows the extracted $D_{i t}$ as a function of energy showing distributions that are consistent with typical $\mathrm{Al}_{2} \mathrm{O}_{3} / \mathrm{Si}$ interfaces and planar reference samples fabricated in the same study. 
The $\mathrm{C}-\mathrm{V}$ measurement also enables the profiling of the majority free carrier density. If the dopant density is high enough $\left(>10^{17} \mathrm{~cm}^{-3}\right)$, free carrier density will not significantly deviate from the dopant density, and therefore, the dopant profile can be calculated. In HF measurements, the depletion capacitance, $C_{d e p}$ can be extracted from the series combination of $C_{o x}$ and $C_{d e p}$. The depletion charge density can then be evaluated from experiment using $Q_{d e p}(V)=\int C_{d e p} d V$, which can lead to a straightforward calculation of the doping density $N_{a}(V)$. Given the cylindrical capacitance, $C_{\text {dep }}(V)$ can be expressed as $C_{d \notin p}=2 \pi \varepsilon \varepsilon_{0} / \ln \left(d / 2 r_{i}\right)$, where $r_{i}$ is the inner radius of the depleted region, one can then extract $r_{i}(V)$ and then calculate $N_{a}(r)$. To account for the change in the population density of the interface traps as the Fermi energy is swept from the valence band-edge to the midgap and consequently the surface potential is changed causing a stretch-out in the $\mathrm{C}-\mathrm{V}$ characteristics, the high-low method can also be used to correct for this stretch out using

$$
N_{a}^{*}(r)=\frac{\left(1-C_{L F} / C_{O x}\right)}{\left(1-C_{H F} / C_{o x}\right)} N_{a}(r)
$$

which is plotted in Figure 17d. The radial dopant distribution is similar to that obtained from modeling and also to reference planar samples. Therefore, the $\mathrm{C}-\mathrm{V}$ profiling is a powerful

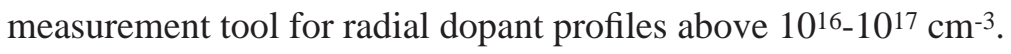


(a)

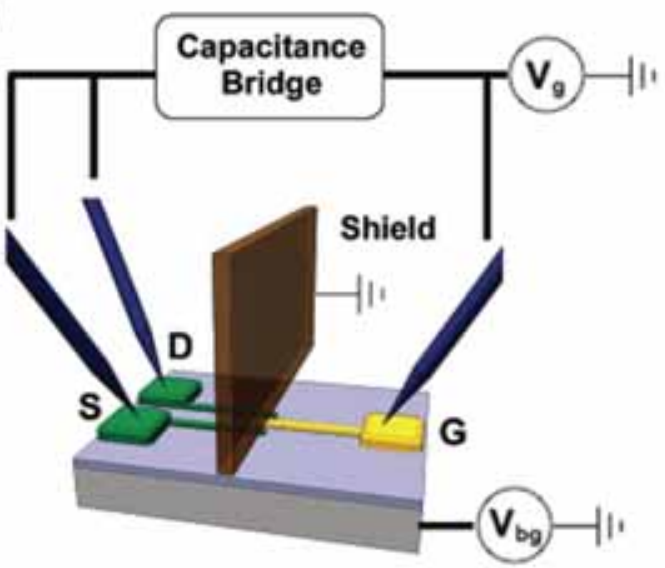

(c)

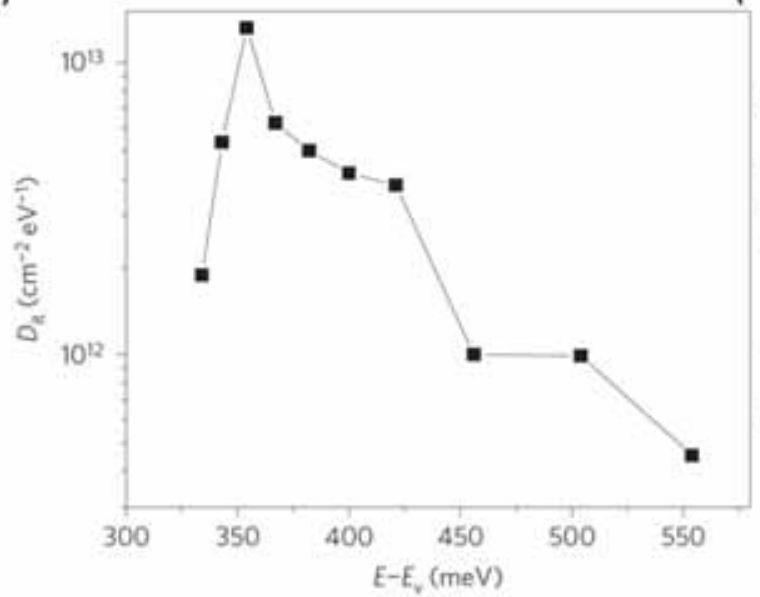

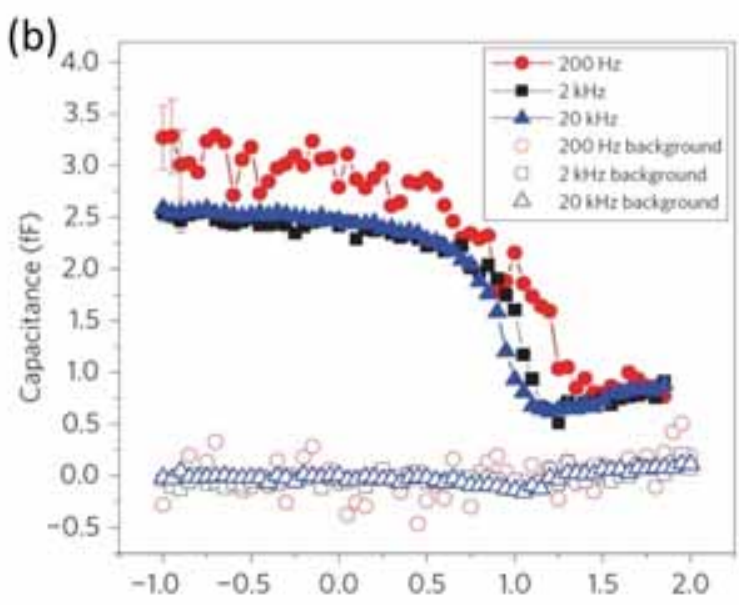

(d)

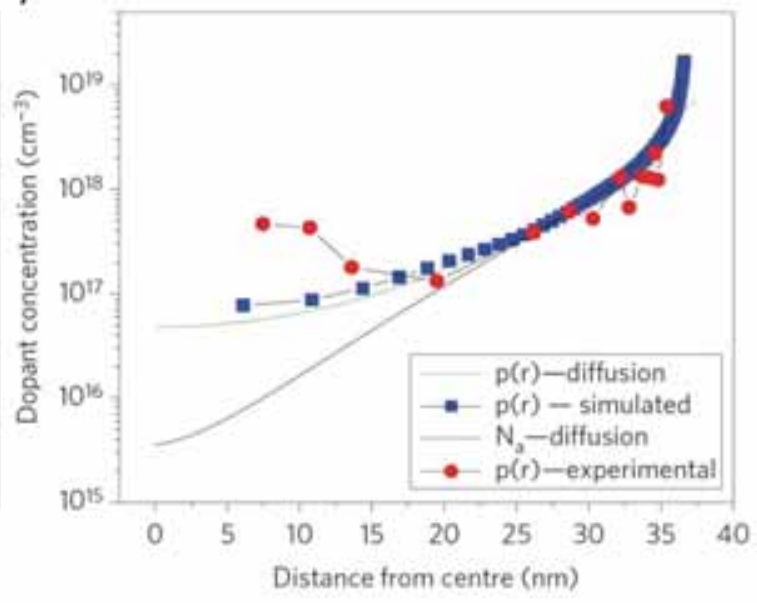

Figure 17: (a) Schematic illustration for the measurement of small NW capacitance. Reproduced with permission from ref. [156]. Copyright American Chemical Society. (b) C-V characterization of a postgrowth B-doped Si NW performed at $77 \mathrm{~K}$ and a $V_{b g}=-20 \mathrm{~V}$; the background capacitance was measured with $V_{b g}=8 \mathrm{~V}$. (c) Extracted interface trap density as a function of energy separation from the valence band-edge. (d) Extracted dopant concentration as a function of radius (red circles) and simulated T-Supreme Boron dopant profile (black) together with calculated carrier concentration from simulated dopant profile (green) and theoretical C-V curve (blue). Reproduced with permission from ref. [157]. Copyright Nature publishing group.

\section{VIII. p-n Junction Formation During Growth and Interface Abruptness:}

Switching dopant gases can result in dopant modulation ${ }^{158,159}$ or p-n junction formation in NWs. ${ }^{13,}$ 97,160,161,162 We briefly overview here two techniques for profiling NW junctions, the scanning capacitance microscopy (SCM) and off-axis electron holography despite the availability of other compelling techniques such as electron-beam induced current (EBIC). ${ }^{163,164}$ The SCM utilizes a high frequency resonator (cantilever with metallized ultrasmall tip diameter) that is sensitive to small changes in capacitance through a shift in the resonant frequency of the measurement circuit. The timedependent signal is fed to a lock-in amplifier with a reference signal that has the same frequency as the signal to be measured, and the output signal is processed to provide a $d C / d V_{\text {signal, which is }}$ 
dependent on the carrier concentration. The actual dopant concentration cannot be measured directly with this technique. Vallett et al. have utilized SCM to characterize doping profiles in p-n+ Si NWs (Figure $18 \mathrm{a}, \mathrm{b}$ ) with $4 \times 10^{-2}$ and $4 \times 10^{-3} \Omega$-cm resistivities and that was subject to an $800^{\circ} \mathrm{C}$ thermal oxidation for 15 minutes. ${ }^{165}$ The metallurgical junction is defined where $d C / d V=0$. Strong change in

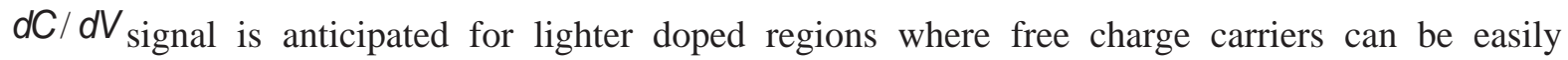
modulated. This is clearly seen in the p-region of Figure 18b. The increase in the $d C / d V_{\text {signal }}$ indicated that the $\mathrm{p}$-doping was graded from $\mathrm{p}$ to $\mathrm{p}$-. The long graded profiles and large depletion region was un-intended for the tunnel FET application that the authors targeted. The long grading profiles were attributed to the thermal oxidation step at $800^{\circ} \mathrm{C}$. Bassani et al. grew axial p-n junction Si NWs while the $\mathrm{HCl}$ flow was maintained during the growth to eliminate tapering and loss of the Au-Si droplet from the NW tip during growth. ${ }^{166}$ This has resulted in a much sharper transition region of $\sim 60 \mathrm{~nm}$ on a $65 \mathrm{~nm}$ diameter $\mathrm{Si} \mathrm{NW}$ as shown in Figure 18c,d. While this is a significant improvement, it demonstrates abrupt junctions in the Si/Ge p-n junctions are not feasible with VLS growth.
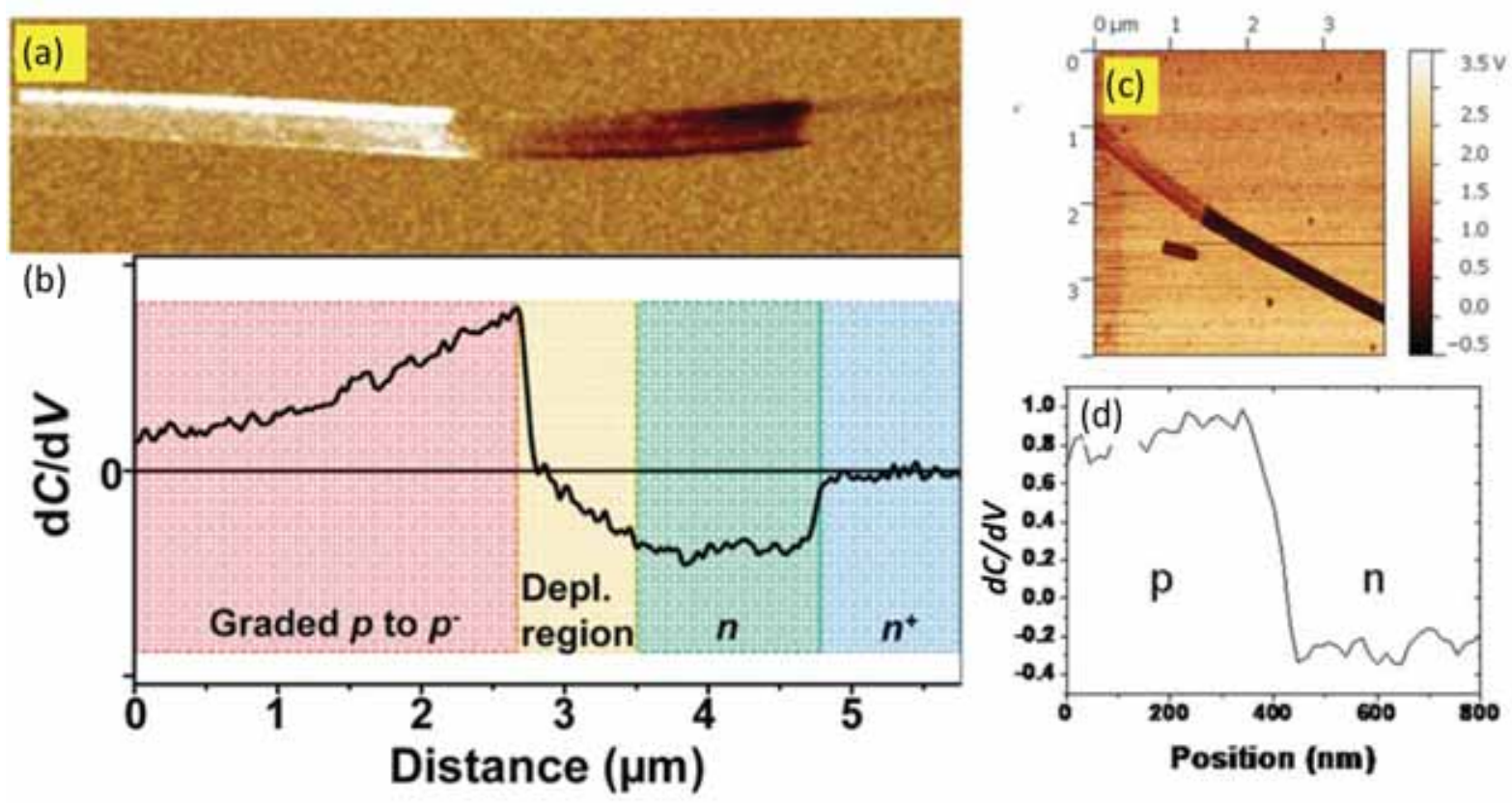

Figure 18: Examples of scanning capacitance microscopy on Si NW p-n junctions. (a) SCM showing $\mathrm{dC} / \mathrm{dV}$ on a $90 \mathrm{~nm}$ diameter $\mathrm{p}-\mathrm{n}+\mathrm{Si} \mathrm{NW}$ with $4 \mathrm{~nm}$ thermally grown $\mathrm{SiO}^{2}$ shell. (b) Line scan of the average $\mathrm{dC} / \mathrm{dV}$ contrast across the width of the NW. Reproduced with Permission from ref. [ $\left.{ }^{165}\right]$. Copyright American Chemical Society. (c) SCM showing dC/dV on a $65 \mathrm{~nm}$ diameter p-n Si NW HCl flow maintained during the growth to prevent shell coating/VS deposition. (e) Line scan of the average $\mathrm{dC} / \mathrm{dV}$ contrast across the width of the NW. Reproduced with permission from ref. [166]. Copyright Wiley-VCH Verlag GmbH \& Co., KGaA, Weinheim.

Off-axis electron holography has also been used to map active dopants in NWs. ${ }^{167}$ Electron holography is a TEM-based interferometric technique where the phase shift of the electron wave is 
caused by the electrostatic and magnetic fields within and surrounding the sample. In the absence of a magnetic field, the phase shift can be expressed as:

$\Delta \varphi(x, y)=C_{E} \int\left(V_{0}(x, y, z)+V_{b i}(x, y, z)\right) d z$

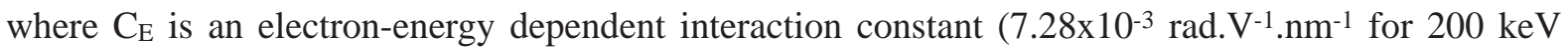
electrons). ${ }^{168}$ Gan et al. utilized the technique to map dopant profiles in CVD grown axial Si NW n-p junction where p-region is remotely doped by surface traps on Si that pin the Fermi energy below midgap. ${ }^{168}$ The graded n-region was found to extend $\sim 60 \mathrm{~nm}$ that is compared to the $80 \mathrm{~nm}$ NW diameter (Figure 19a,b). Darbandi et al. measured holograms on OMVPE grown GaAs NWs growth with tertiarybutylarsine as the group $\mathrm{V}$ precursor and diethylzinc and diethlyeltellurium as the $\mathrm{p}$ $\left(6 \times 10^{19} \mathrm{~cm}^{-3}\right)$ and n-type $\left(5 \times 10^{17} \mathrm{~cm}^{-3}\right)$ precursors, respectively. ${ }^{169}$ The measured depletion regions were $39 \mathrm{~nm}$ in the n-region and $35 \mathrm{~nm}$ in the p-region (Figure 19). The calculated depletion region from the dopant concentrations is $64 \mathrm{~nm}$ predominantly in the n-type lower doped segment of the device. The result agrees quantitatively with the measured depletion width but the extracted location of metallurgical junction was shifted. Nonetheless, the authors here have succeeded in the formation of an abrupt GaAs NW p-n junction. 

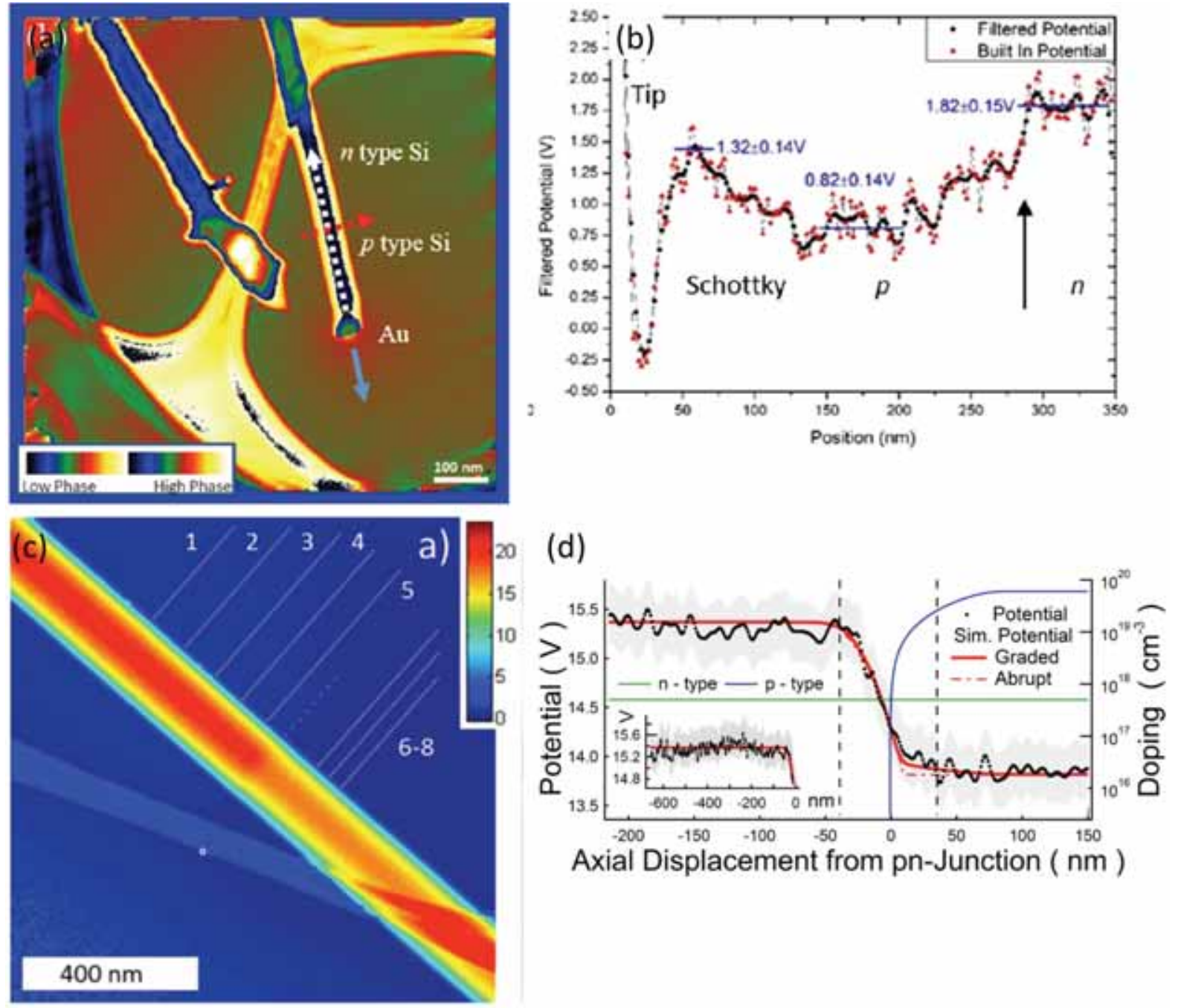

Figure 19: Example off-axis electron holography to map junction profiles in NWs. (a) Hologram of an 80nm diameter Si NW n-p (p-type due to surface traps) junction and (b) potential calculated from the phase shift in (a). Reproduced with permission from ref. [168]. Copyright American Institute of Physics. (c) Hologram of a GaAs NW p-n junction and (d) potential calculated from phase-shift in (c). Reproduced with permission from ref. [169]. Copyright American Chemical society.

\section{Limits of Nanowire Doping}

There are many physical origins for limiting dopant incorporation and ionization in NWs. The thermodynamic limits on doping include dopant solubility limits and size-induced dopant segregation. The electrical limits on doping include increased ionization energies and quantum confinement effects. The dopant solid solubility sets a limit for dopant incorporation in NWs. Schmid et al. grew multiple Si NWs with different $\mathrm{PH}_{3} / \mathrm{SiH}_{4}$ ratios and found that the doping concentration saturated at $1.5 \times 10^{20} \mathrm{~cm}^{-3}$ when $\mathrm{PH}_{3} / \mathrm{SiH}_{4}$ ratio was $1.5 \times 10^{-2} .50$ Higher $\mathrm{PH}_{3} / \mathrm{SiH}_{4}$ ratio did not increase the doping concentration, which was attributed to the solid solubility limit of P in Si. 
Size-induced surface segregation of dopants is another limiting factor in NW doping. Dalpian et al. used first principles methods to show that the formation energy of impurities in nanocrystals increases as the size of nanocrystals decreases, creating deep impurity level in the gap of nanocrystals. ${ }^{170}$ Thus more energy is required for doping nanostructures compared to bulk and this intrinsic behavior of nanostructure was termed as "self-purification". ${ }^{170} \mathrm{By}$ means of $a b$ initio simulations, Fernández-Serra et al. ${ }^{66}$ studied boron and phosphorus segregation in unpassivated and passivated Si NWs. In unpassivated NWs, impurities tend to segregate at the surface whereas the segregation energy in passivated NWs was found to be larger than that of unpassivated surfaces. The impurities segregate to passivate the surface dangling bonds where they become electronically inactive. The role of surface dangling bonds and their passivation in doping NWs was extensively studied by many other groups. $67,171-175$

For bulk semiconductors, the Coulomb potential is screened and impurities are readily ionized. However, when it comes to NWs, ionization energy of dopant impurities is enhanced at small diameters, a separate effect from quantum confinement. ${ }^{176}$ This is due to dielectric confinement which originates from the dielectric mismatch between the NW and its surroundings. Diarra et al. ${ }^{176}$ set up an analytical tight binding (TB) model deduced below equation for the ionization energy

$$
E_{l}-E_{l}^{o} \cong \frac{2 q^{2}}{\varepsilon_{\text {in }} R} \frac{\varepsilon_{\text {in }}-\varepsilon_{\text {out }}}{\varepsilon_{\text {in }}+\varepsilon_{\text {out }}} F\left(\frac{\varepsilon_{\text {in }}}{\varepsilon_{\text {out }}}\right) \propto 1 / d
$$

where and is the ionization energy of donor impurities in NW and bulk, respectively, $q$ is the fundamental charge constant, $\varepsilon_{\text {in }}$ and $\varepsilon_{\text {out }}$ are the dielectric constants for the NW and its surrounding medium, respectively, and $F(x)$ is a positive function independent of wire diameter. Equation (13) demonstrates that the ionization energy is strongly affected by dielectric constants and is inversely proportional to the size of NW.

This effect of dielectric confinement in doping was experimentally confirmed by Bjork et al. ${ }^{177}$ who studied the resistivity of VLS grown Si NWs with different diameters and different nominal doping concentrations. They found that the resistivity increased as the diameter decreased for NWs grown at the same nominal doping concentration as shown in Figure 20. This is due to donor deactivation in Si NWs, which originates from dielectric mismatch between NW and its surroundings. When a NW is surrounded by a low dielectric 
constant medium, the screening of the impurity potential decreases compared to the bulk semiconductor case, which results in an increase in the donor ionization energy and this dielectric confinement gets stronger as the diameter of NW decreases, as discussed above. ${ }^{176}$ The increase in resistivity as a function of decreasing NW diameter was suppressed for NWs coated with $\mathrm{Al}_{2} \mathrm{O}_{3}$, which has a smaller dielectric mismatch with NWs compared to that of NWs surrounded by air as shown in Figure 20d.
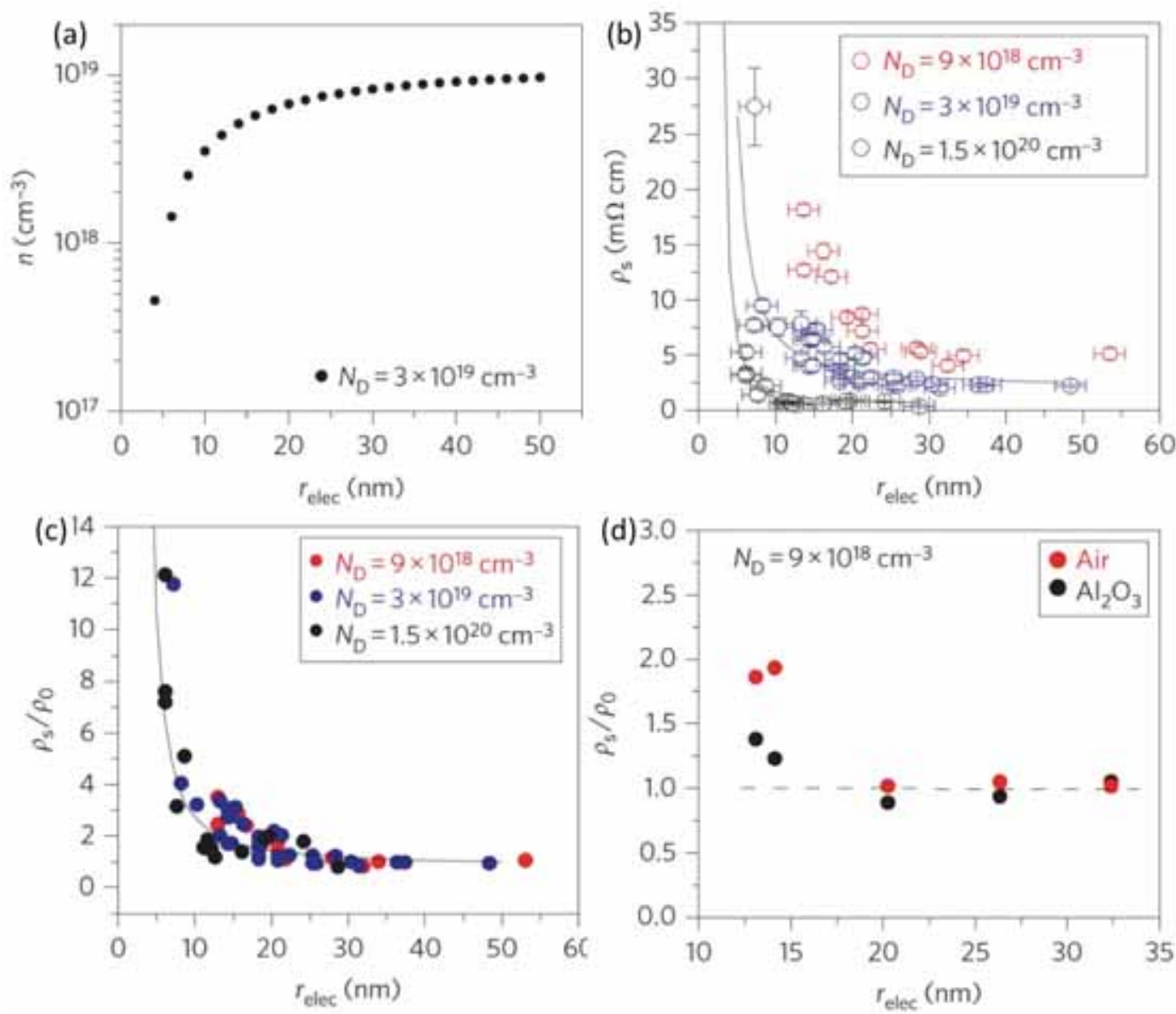

Figure 20: (a) Calculated electron concentration in the conduction band as a function of radius for . (b) Measured resistivity as a function of radius. The solid curves correspond to the calculated resistivity due to a changing donor ionization energy obtained using the data in (a) for and similarly for and . (c) Normalized resistivity (measured resistivity, divided by resistivity of the largest radii wire of each doping series) as a function of electronic radius for measured data and for the theoretical calculation (solid line). (d) Normalized resistivity versus radius for non-coated and $\mathrm{Al}_{2} \mathrm{O}_{3}$-coated $\mathrm{NWs}$ (). Reproduced with permission from ref. [177]. Copyright Nature Publishing Company.

Quantum confinement can also limit free carrier concentration in NWs. ${ }^{178}$ The compensation of external dopants with native defects is explained by the amphoteric defect model. ${ }^{179}$ With extrinsic doping, the Fermi level () moves away from Fermi stabilization energy () but consequently, the generation of amphoteric native defects gets favorable and 
compensate the external dopants so that moves back toward and saturates at a limit value . 179 The NW density of states (1D DOS) for a single valley in the band structure can be expressed as

$$
\rho^{1 D}(E)=\frac{1}{\pi \hbar a^{2}} \sum_{N_{x}, N_{y}} \sqrt{\frac{2 m_{z}^{*}}{E-\left(E_{0}+E_{N_{x} N_{y}}\right)}}
$$

where $E_{0}$ is $E_{c}$ for n-type and $E_{v}$ for p-type doping and is the effective mass of carriers along the NW length direction, $E_{N x, N_{y}}=\hbar^{2} \pi^{2}\left(N_{x}^{2}+N_{y}^{2}\right) / 2 m_{x y}^{*} a^{2}$ is the quantized energy level, and $m_{x y}^{*}$ is the effective mass in $(x, y)$ plane. By integration of the 1D DOS from $E=E_{0}$ to $E=E_{F-\text { limit }}$, the maximum achievable carrier concentration in terms of the NW width was obtained as shown in Figure 21. This demonstrates the limitation of doping for small NWs due to the quantum confinement effect.

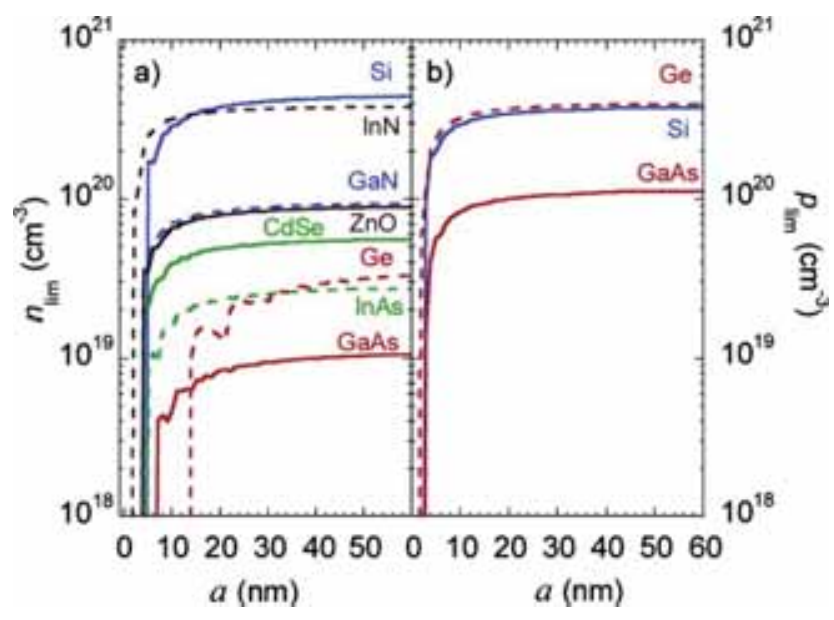

Figure 21: Calculated maximum achievable electron (a) and hole (b) concentrations in various semiconductor NWs as a function of NW width a. Reproduced with permission from ref. [178]. Copyright American Chemical Society.

In compound semiconductor NWs, dopant compensation can also limit NW doping. Hilse et al. doped GaAs NWs with $\mathrm{Si}$ or $\mathrm{Be}$ at different growth temperatures and characterized them with Raman spectroscopy. ${ }^{180}$ GaAs NWs with higher nominally Be doping showed a previously unobserved Raman peak at $384 \mathrm{~cm}^{-1}$ that was attributed to Be incorporation at interstitial sites. For the Si doped GaAs NWs, Raman peaks of $\mathrm{Si}_{\mathrm{Ga}}, \mathrm{Si}_{\mathrm{As}}$ and $\mathrm{Si}_{\mathrm{Ga}}-\mathrm{Si}_{\mathrm{As}}$ pairs were detected indicating that $\mathrm{Si}$ was incorporated into $\mathrm{GaAs}$ as both a donor and an acceptor. Thus self-compensation of $\mathrm{Si}_{\mathrm{Ga}}$ donors and $\mathrm{Si}_{\mathrm{As}}$ acceptors occurs during doping. Compensation mechanism of Si dopants to GaAs was further studied by Ketterer et 
al. Catalyst-free GaAs NWs doped by Si with different Si fluxes in MBE followed by Raman spectroscopy. ${ }^{181}$ The Raman spectra results also corroborated the presence of $\mathrm{Si}_{\mathrm{Ga}}-\mathrm{Si}_{\mathrm{As}}$ pair sites at higher $\mathrm{Si}$ fluxes in addition to the expected $\mathrm{Si}_{\mathrm{As}}$ peaks. Positively charged $\mathrm{Si}$ Ga donors and negatively charged $\mathrm{Si}_{\mathrm{As}}$ acceptors compensate each other when their separation is similar or smaller than the screening length leading to the formation of a neutral Si pair and electrical deactivation of Si atoms. ${ }^{106}$

\section{Conclusions}

While extensive research efforts in NW growth and doping have significantly advanced our understanding of and control over in-situ doping during growth, multiple challenging aspects are yet to be overcome:

- Doping gradients and junctions: The complex faceted geometry of NWs co-existing with a triplephase boundary at the droplet-NW interface leads to radial gradients in impurity doping in the liquid droplet and in the NW crystal. It might be possible to balance or engineer these radial gradients with alloyed liquid droplets. It might also be possible to tailor the chemical potentials and doping gradients by applying electric fields that might impose equipotential conditions at the liquid-solid interface as long as supersaturation requirements do not demand growth conditions that degrade the NW morphology. Edge effects that are governed by surface energies however may not be fully controlled during seeded or unseeded NW growth. The overgrowth of doped layers may help to deterministically control the radial dopant profiles but cannot be applied where ultra narrow NW diameters are desired. Additionally, the reservoir effects lead to axial doping gradients, which limit the control over junction formation. In elemental NWs, alloyed droplets helped sharpening heterojunction profiles, ${ }^{182}$ but the stability of the droplet was compromised and surface contamination from droplet diffusion on the NW sidewalls evolved. The elimination of doping gradients remains a significant challenge for VLS NWs.

- Morphology and crystal control: Complex precursor and heterogeneous gas-solid reactions can compromise the resulting NW morphology. Recent progress in utilizing simultaneous growth and sidewall etching (e.g. $\mathrm{HCl}$ ) during growth helped mitigate this problem. Extension of this technique to a wide range of NW materials may be faced with compatibility challenges. Polytypic growth of Wurtzite and Zinc Blende emerges in alternate doped III-V NWs, and phase purity in doped NWs is yet to be achieved without compromising other factors such as morphology and doping levels.

- Droplet impurity contamination: There is compelling evidence that significant amounts of droplet impurity incorporate in the grown semiconductor NW material. Alternative catalysts than Au, such as $\mathrm{Cu}$ and Al may have lower distribution coefficients in the solid NW that is yet to be proven with the techniques (SIMS, LEAP, STEM) used to quantify Au incorporation. NW growths that do not utilize a 
metal growth seed such as self-catalyzed, selective area, and oxide-assisted growth, eliminate this problem. Their application however is limited to certain materials such as III-V and II-VI where phase purity and dopant control is yet to be demonstrated.

- Thermodynamic and confinement limits: Dopant segregation to the surface can also impede progress in obtaining uniformly doped NWs. Early results on in-situ surface passivation are promising and need to be extended to very small diameter NWs. At such scales, other challenges such as quantum confinement can limit the electrically active free-carrier density in NWs. At high doping levels, some III-V dopants exhibit strong amphoteric doping characteristics putting a bound on the maximum achievable dopant density.

We believe that focused efforts in these areas can lead to the sought objective of full control over the doping density and profiles without compromising other NW characteristics. Our objective is to promote additional focus on these challenges rather than pointing at them as unsolvable issues. We believe that the progress thus far in addressing some of these problems that are overviewed in this manuscript is exceptional and holds great promise for this unique class of materials.

Acknowledgments: This work was supported by the LDRD program at Los Alamos National Laboratory, the National Science Foundation (DMR-1503595, NSF CAREER Award ECCS-1351980) and an Office of Naval Research Multidisciplinary University Research Initiative (ONR MURI) award N00014-13-1-0678. This work was performed, in part, at the Center for Integrated Nanotechnologies, an Office of Science User Facility operated for the U.S. Department of Energy (DOE) Office of Science. Sandia National Laboratories is a multi-program laboratory managed and operated by Sandia Corporation, a wholly owned subsidiary of Lockheed Martin Corporation, for the U.S. Department of Energy's National Nuclear Security Administration under contract DEAC04-94AL85000.

\section{References:}

1 Wagner, R. \& Ellis, W. Vapor- liquid- solid mechanism of single crystal growth. Applied Physics Letters 4, 89-90 (1964).

2 Givargizov, E. Fundamental aspects of VLS growth. Journal of Crystal Growth 31, 20-30 (1975).

3 Bootsma, G. \& Gassen, H. A quantitative study on the growth of silicon whiskers from silane and germanium whiskers from germane. Journal of Crystal Growth 10, 223-234 (1971).

4 Kaldis, E. \& Scheel, H. J. Current Topics in Materials Science. (North-Holland Publishing Company, 1977).

5 Kasahara, J., Kajiwara, K. \& Yamada, T. GaAs whiskers grown by a thermal decomposition method. Journal of Crystal Growth 38, 23-28 (1977).

6 Noras, J. \& Ryall, M. Whisker growth on nickel-coated gallium phosphide. Journal of Physics D: Applied Physics 12, 277 (1979). 
7 Givargizov, E. I. Oriented growth of whiskers of AIIIBV compounds by VLSmechanism. Kristall und Technik 10, 472 (1975).

8 Wolfe, C., Nuese, C. \& Holonyak Jr, N. Growth and Dislocation Structure of SingleCrystal Ga (As1- xPx). Journal of Applied Physics 36, 3790-3801 (1965).

9 Haraguchi, K., Katsuyama, T., Hiruma, K. \& Ogawa, K. GaAs p- n junction formed in quantum wire crystals. Applied Physics Letters 60, 745-747 (1992).

10 Morales, A. M. \& Lieber, C. M. A laser ablation method for the synthesis of crystalline semiconductor nanowires. Science 279, 208-211 (1998).

$11 \mathrm{Wu}$, Y., Fan, R. \& Yang, P. Block-by-block growth of single-crystalline Si/SiGe superlattice nanowires. Nano Letters 2, 83-86 (2002).

12 Björk, M. et al. One-dimensional steeplechase for electrons realized. Nano Letters 2 , 87-89 (2002).

13 Gudiksen, M. S., Lauhon, L. J., Wang, J., Smith, D. C. \& Lieber, C. M. Growth of nanowire superlattice structures for nanoscale photonics and electronics. Nature $\mathbf{4 1 5}$, 617-620 (2002).

14 Lauhon, L. J., Gudiksen, M. S., Wang, D. \& Lieber, C. M. Epitaxial core-shell and coremultishell nanowire heterostructures. Nature 420, 57-61 (2002).

15 Gudiksen, M. S., Wang, J. \& Lieber, C. M. Size-dependent photoluminescence from single indium phosphide nanowires. The Journal of Physical Chemistry B 106, 4036-4039 (2002).

16 Trägårdh, J., Persson, A., Wagner, J., Hessman, D. \& Samuelson, L. Measurements of the band gap of wurtzite InAs1- xPx nanowires using photocurrent spectroscopy. Journal of applied physics 101, 123701 (2007).

17 Joyce, H. J., Wong-Leung, J., Gao, Q., Tan, H. H. \& Jagadish, C. Phase perfection in zinc blende and wurtzite III- V nanowires using basic growth parameters. Nano letters $\mathbf{1 0}$, 908-915 (2010).

18 Caroff, P. et al. Controlled polytypic and twin-plane superlattices in III-V nanowires. Nature nanotechnology 4, 50-55 (2009).

19 Bakkers, E. P. \& Verheijen, M. A. Synthesis of InP nanotubes. Journal of the American Chemical Society 125, 3440-3441 (2003).

20 Thelander, C. et al. Nanowire-based one-dimensional electronics. Materials today $\mathbf{9}$, 28-35 (2006).

21 Pauzauskie, P. J. \& Yang, P. Nanowire photonics. Materials Today 9, 36-45 (2006).

22 Hochbaum, A. I. et al. Enhanced thermoelectric performance of rough silicon nanowires. Nature 451, 163-167 (2008).

23 McAlpine, M. C., Ahmad, H., Wang, D. \& Heath, J. R. Highly ordered nanowire arrays on plastic substrates for ultrasensitive flexible chemical sensors. Nature materials $\mathbf{6}$, 379-384 (2007).

24 Patolsky, F., Timko, B. P., Zheng, G. \& Lieber, C. M. Nanowire-based nanoelectronic devices in the life sciences. MRS bulletin 32, 142-149 (2007).

25 Patolsky, F. et al. Detection, stimulation, and inhibition of neuronal signals with highdensity nanowire transistor arrays. Science 313, 1100-1104 (2006).

26 Yazawa, M., Koguchi, M., Muto, A. \& Hiruma, K. Semiconductor nanowhiskers. Advanced Materials 5, 577-580 (1993). 
27 Dick, K. A. et al. A New Understanding of Au- Assisted Growth of III-V Semiconductor Nanowires. Advanced Functional Materials 15, 1603-1610 (2005).

28 Novotny, C. J. \& Paul, K. Vertically aligned, catalyst-free InP nanowires grown by metalorganic chemical vapor deposition. Applied Physics Letters 87, 203111 (2005).

29 Motohisa, J., Noborisaka, J., Takeda, J., Inari, M. \& Fukui, T. Catalyst-free selective-area MOVPE of semiconductor nanowires on (111) B oriented substrates. Journal of crystal growth 272, 180-185 (2004).

30 Dubrovskii, V. et al. Diffusion-induced growth of GaAs nanowhiskers during molecular beam epitaxy: Theory and experiment. Physical review B 71, 205325 (2005).

31 Jensen, L. E. et al. Role of surface diffusion in chemical beam epitaxy of InAs nanowires. Nano Letters 4, 1961-1964 (2004).

32 Zhi, C., Bai, X. \& Wang, E. Synthesis of semiconductor nanowires by annealing. Applied physics letters 85 (2004).

33 Park, H. D., Prokes, S. M. \& Cammarata, R. C. Growth of epitaxial InAs nanowires in a simple closed system. Applied Physics Letters 87, 063110 (2005).

34 Duan, X., Wang, J. \& Lieber, C. M. Synthesis and optical properties of gallium arsenide nanowires. Applied Physics Letters 76, 1116-1118 (2000).

35 Shi, W., Zheng, Y., Wang, N., Lee, C. \& Lee, S. Oxide-assisted growth and optical characterization of gallium-arsenide nanowires. Applied Physics Letters 78, 3304-3306 (2001).

$36 \mathrm{Xu}, \mathrm{X}$. et al. Synthesis of InAs nanowires via a low-temperature solvothermal route. Nanotechnology 17, 3416 (2006).

37 Dayeh, S. A., Yu, E. T. \& Wang, D. Excess indium and substrate effects on the growth of InAs nanowires. small 3, 1683-1687 (2007).

38 Laocharoensuk, R. et al. Flow-based solution-liquid-solid nanowire synthesis. Nature nanotechnology 8, 660-666 (2013).

39 Park, H. D., Prokes, S., Twigg, M., Cammarata, R. C. \& Gaillot, A.-C. Si-assisted growth of InAs nanowires. (DTIC Document, 2006).

40 Bierman, M. J., Lau, Y. A., Kvit, A. V., Schmitt, A. L. \& Jin, S. Dislocation-driven nanowire growth and Eshelby twist. Science 320, 1060-1063 (2008).

41 Fröberg, L., Seifert, W. \& Johansson, J. Diameter-dependent growth rate of InAs nanowires. Physical Review B 76, 153401 (2007).

42 Sutter, E. A. \& Sutter, P. W. Size-Dependent Phase Diagram of Nanoscale Alloy Drops Used in Vapor- Liquid- Solid Growth of Semiconductor Nanowires. ACS nano 4, 4943-4947 (2010).

43 Schmidt, V., Senz, S. \& Gösele, U. The shape of epitaxially grown silicon nanowires and the influence of line tension. Applied Physics A 80, 445-450 (2005).

44 Naidich, Y. V., Perevertailo, V. \& Obushchak, L. Contact properties of the phases participating in the crystallization of gold-silicon and gold-germanium melts. Powder Metallurgy and Metal Ceramics 14, 567-571 (1975).

45 Sutter, E. \& Sutter, P. Phase diagram of nanoscale alloy particles used for vapor-liquidsolid growth of semiconductor nanowires. Nano letters 8, 411-414 (2008).

46 Gamalski, A., Tersoff, J., Sharma, R., Ducati, C. \& Hofmann, S. Formation of metastable liquid catalyst during subeutectic growth of germanium nanowires. Nano letters $\mathbf{1 0}$, 2972-2976 (2010). 
47 Dayeh, S. A. \& Picraux, S. T. Direct Observation of Nanoscale Size Effects in Ge Semiconductor Nanowire Growth. Nano Letters 10, 4032-4039, doi:10.1021/nl1019722 (2010).

48 Schwalbach, E. J. \& Voorhees, P. W. Doping nanowires grown by the vapor-liquid-solid mechanism. Applied Physics Letters 95, 063105, doi:doi:http://dx.doi.org/ 10.1063/1.3204543 (2009).

49 Sivaram, S. V., Shin, N., Chou, L.-W. \& Filler, M. A. Direct Observation of Transient Surface Species during Ge Nanowire Growth and Their Influence on Growth Stability. Journal of the American Chemical Society 137, 9861-9869, doi:10.1021/jacs.5b03818 (2015).

50 Schmid, H. et al. Doping limits of grown in situ doped silicon nanowires using phosphine. Nano Letters 9, 173-177 (2008).

51 Schmid, H. et al. Patterned epitaxial vapor-liquid-solid growth of silicon nanowires on $\mathrm{Si}$ (111) using silane. Journal of Applied Physics 103, 024304 (2008).

52 Nimmatoori, P., Zhang, Q., Dickey, E. C. \& Redwing, J. M. Suppression of the vaporliquid-solid growth of silicon nanowires by antimony addition. Nanotechnology $\mathbf{2 0}$, 025607 (2008).

53 Perea, D. E. et al. Direct measurement of dopant distribution in an individual vapourliquid-solid nanowire. Nature Nanotechnology 4, 315-319 (2009).

54 Glas, F., Harmand, J.-C. \& Patriarche, G. Why does wurtzite form in nanowires of III-V zinc blende semiconductors? Physical review letters 99, 146101 (2007).

55 Gamalski, A., Ducati, C. \& Hofmann, S. Cyclic supersaturation and triple phase boundary dynamics in germanium nanowire growth. The Journal of Physical Chemistry C 115, 4413-4417 (2011).

$56 \mathrm{Oh}, \mathrm{S}$. H. et al. Oscillatory mass transport in vapor-liquid-solid growth of sapphire nanowires. Science 330, 489-493 (2010).

57 Wen, C.-Y. et al. Periodically changing morphology of the growth interface in $\mathrm{Si}, \mathrm{Ge}$, and GaP nanowires. Physical review letters 107, 025503 (2011).

58 Schwalbach, E. \& Voorhees, P. Doping nanowires grown by the vapor-liquid-solid mechanism. Applied Physics Letters 95, 063105 (2009).

59 Connell, J. G. et al. Identification of an intrinsic source of doping inhomogeneity in vapor-liquid-solid-grown nanowires. Nano letters 13, 199-206 (2012).

60 Saul, R. \& Hackett, W. Distribution of Impurities in Zn, O- Doped GaP Liquid Phase Epitaxy Layers. Journal of The Electrochemical Society 117, 921-924 (1970).

61 Clark, T. E. et al. Diameter Dependent Growth Rate and Interfacial Abruptness in VaporLiquid-Solid Si/Si1- x Ge x Heterostructure Nanowires. Nano letters 8, 1246-1252 (2008).

62 Li, N., Tan, T. Y. \& Gösele, U. Transition region width of nanowire hetero-and pnjunctions grown using vapor-liquid-solid processes. Applied Physics A 90, 591-596 (2008).

63 Picraux, S. T., Dayeh, S. A., Manandhar, P., Perea, D. E. \& Choi, S. G. Silicon and germanium nanowires: Growth, properties, and integration. Jom 62, 35-43 (2010).

64 Koren, E., Rosenwaks, Y., Allen, J., Hemesath, E. \& Lauhon, L. Nonuniform doping distribution along silicon nanowires measured by Kelvin probe force microscopy and scanning photocurrent microscopy. Applied Physics Letters 95, 092105 (2009). 
$65 \mathrm{Ou}, \mathrm{X}$. et al. Carrier profiling of individual Si nanowires by scanning spreading resistance microscopy. Nano letters 10, 171-175 (2009).

66 Fernández-Serra, M., Adessi, C. \& Blase, X. Surface segregation and backscattering in doped silicon nanowires. Physical review letters 96, 166805 (2006).

67 Peelaers, H., Partoens, B. \& Peeters, F. M. Formation and segregation energies of B and P doped and BP codoped silicon nanowires. Nano letters 6, 2781-2784 (2006).

68 Xie, P., Hu, Y., Fang, Y., Huang, J. \& Lieber, C. M. Diameter-dependent dopant location in silicon and germanium nanowires. Proceedings of the National Academy of Sciences 106, 15254-15258 (2009).

69 Givargizov, E. \& Sheftal, N. Morphology of Germanium Whiskers. Kristall und Technik 7, 37-41 (1972).

70 Givargizov, E. Periodic instability in whisker growth. Journal of Crystal Growth 20, 217-226 (1973).

71 Schwarz, K. \& Tersoff, J. From droplets to nanowires: Dynamics of vapor-liquid-solid growth. Physical review letters 102, 206101 (2009).

72 Dayeh, S. A. et al. Growth, defect formation, and morphology control of germaniumsilicon semiconductor nanowire heterostructures. Nano letters 11, 4200-4206 (2011).

73 Jeon, N., Dayeh, S. A. \& Lauhon, L. J. Origin of polytype formation in VLS-grown Ge nanowires through defect generation and nanowire kinking. Nano letters 13, 3947-3952 (2013).

74 Musin, I. R. \& Filler, M. A. Chemical control of semiconductor nanowire kinking and superstructure. Nano letters 12, 3363-3368 (2012).

75 Zheng, G., Lu, W., Jin, S. \& Lieber, C. M. Synthesis and fabrication of highperformance n- type silicon nanowire transistors. Advanced Materials 16, 1890-1893 (2004).

76 Wang, Y. et al. Use of phosphine as an n-type dopant source for vapor-liquid-solid growth of silicon nanowires. Nano letters 5, 2139-2143 (2005).

77 Lew, K.-K. et al. Structural and electrical properties of trimethylboron-doped silicon nanowires. Applied Physics Letters 85, 3101-3103 (2004).

78 Tutuc, E., Guha, S. \& Chu, J. Morphology of germanium nanowires grown in presence of B2H6. Applied physics letters 88, 3113 (2006).

79 Li, F., Nellist, P. D. \& Cockayne, D. J. Doping-dependent nanofaceting on silicon nanowire surfaces. Applied Physics Letters 94, 3111 (2009).

80 Bragg, J. K., McCarty, L. V. \& Norton, F. Kinetics of Pyrolysis of Diborane1. Journal of the American Chemical Society 73, 2134-2140 (1951).

81 Mehta, B. \& Tao, M. A kinetic model for boron and phosphorus doping in silicon epitaxy by CVD. Journal of The Electrochemical Society 152, G309-G315 (2005).

82 Tutuc, E., Chu, J., Ott, J. \& Guha, S. Doping of germanium nanowires grown in presence of PH3. Applied physics letters 89, 3101 (2006).

83 Johansson, J. et al. Structural properties of $\langle 111\rangle$ B-oriented III-V nanowires. Nature materials 5, 574-580 (2006).

84 Ross, F., Tersoff, J. \& Reuter, M. Sawtooth faceting in silicon nanowires. Physical review letters 95, 146104 (2005). 
85 Uesawa, N., Inasawa, S., Tsuji, Y. \& Yamaguchi, Y. Gas-phase synthesis of rough silicon nanowires via the zinc reduction of silicon tetrachloride. The Journal of Physical Chemistry C 114, 4291-4296 (2010).

$86 \mathrm{Xu}, \mathrm{T}$. et al. Faceted sidewalls of silicon nanowires: Au-induced structural reconstructions and electronic properties. Physical Review B 81, 115403 (2010).

87 White, R., Espino- Rios, R., Rogers, D., Ring, M. \& O'neal, H. Mechanism of the silane decomposition. I. Silane loss kinetics and rate inhibition by hydrogen. II. Modeling of the silane decomposition (all stages of reaction). International journal of chemical kinetics 17, 1029-1065 (1985).

88 Jasinski, J. M. \& Gates, S. M. Silicon chemical vapor deposition one step at a time: fundamental studies of silicon hydride chemistry. Accounts of chemical research 24, 9-15 (1991).

89 Dayeh, S. A., Yu, E. T. \& Wang, D. Surface diffusion and substrate- nanowire adatom exchange in InAs nanowire growth. Nano letters 9, 1967-1972 (2009).

90 Dick, K. A. A review of nanowire growth promoted by alloys and non-alloying elements with emphasis on Au-assisted III-V nanowires. Progress in Crystal Growth and Characterization of Materials 54, 138-173 (2008).

91 Jie, J., Zhang, W., Bello, I., Lee, C.-S. \& Lee, S.-T. One-dimensional II-VI nanostructures: synthesis, properties and optoelectronic applications. Nano Today $\mathbf{5}$, 313-336 (2010).

92 Dai, X., Olivier, A., Wilhelm, C., Dayeh, S. \& Soci, C. Advanced III-V nanowire growth toward large-scale integration. Semiconductor Nanowires: Materials, Synthesis, Characterization and Applications, 71 (2015).

93 Dayeh, S. A., Yu, E. T. \& Wang, D. III-V nanowire growth mechanism: V/III ratio and temperature effects. Nano letters 7, 2486-2490 (2007).

94 Dayeh, S. A., Soci, C., Bao, X.-Y. \& Wang, D. Advances in the synthesis of InAs and GaAs nanowires for electronic applications. Nano Today 4, 347-358 (2009).

95 Borgström, M. T. et al. In situ etching for total control over axial and radial nanowire growth. Nano Research 3, 264-270 (2010).

96 Wallentin, J. et al. InP nanowire array solar cells achieving $13.8 \%$ efficiency by exceeding the ray optics limit. Science 339, 1057-1060 (2013).

97 Heurlin, M. et al. Axial InP nanowire tandem junction grown on a silicon substrate. Nano letters 11, 2028-2031 (2011).

98 Wallentin, J. et al. Probing the wurtzite conduction band structure using state filling in highly doped InP nanowires. Nano letters 11, 2286-2290 (2011).

99 Wallentin, J., Ek, M., Wallenberg, L. R., Samuelson, L. \& Borgström, M. T. Electron trapping in InP nanowire FETs with stacking faults. Nano letters 12, 151-155 (2011).

100 Wallentin, J. \& Borgström, M. T. Doping of semiconductor nanowires. Journal of Materials Research 26, 2142-2156, doi:doi:10.1557/jmr.2011.214 (2011).

101 Logan, R., Tanbun- Ek, T. \& Sergent, A. Doping of InP and GaInAs with S during metalorganic vapor- phase epitaxy. Journal of Applied Physics 65, 3723-3725 (1989).

102 Dufouleur, J. et al. P-doping mechanisms in catalyst-free gallium arsenide nanowires. Nano letters 10, 1734-1740 (2010).

103 Stringfellow, G. The role of impurities in III/V semiconductors grown by organometallic vapor phase epitaxy. Journal of Crystal Growth 75, 91-100 (1986). 
104 Gutsche, C. et al. n-type doping of vapor-liquid-solid grown GaAs nanowires. Nanoscale Res. Lett 6, 65 (2011).

105 Spitzer, W. \& Panish, M. Silicon- Doped Gallium Arsenide Grown from Gallium Solution: Silicon Site Distribution. Journal of Applied Physics 40, 4200-4202 (1969).

106 Domke, C., Ebert, P., Heinrich, M. \& Urban, K. Microscopic identification of the compensation mechanisms in Si-doped GaAs. Physical Review B 54, 10288 (1996).

107 Newman, R. The lattice locations of silicon impurities in GaAs: effects due to stoichiometry, the Fermi energy, the solubility limit and DX behaviour. Semiconductor science and technology 9, 1749 (1994).

108 Colombo, C., Heiß, M., Grätzel, M. \& Fontcuberta i Morral, A. Gallium arsenide p-i-n radial structures for photovoltaic applications. Applied Physics Letters 94, 173108, doi:doi:http://dx.doi.org/10.1063/1.3125435 (2009).

109 Borgström, M. T. et al. Precursor evaluation for in situ InP nanowire doping. Nanotechnology 19, 445602 (2008).

110 Wallentin, J. et al. Changes in contact angle of seed particle correlated with increased zincblende formation in doped InP nanowires. Nano letters 10, 4807-4812 (2010).

111 Wallentin, J. et al. Degenerate p-doping of InP nanowires for large area tunnel diodes. Applied Physics Letters 99, 253105 (2011).

112 Rigutti, L. et al. Si incorporation in InP nanowires grown by Au-assisted molecular beam epitaxy. Journal of Nanomaterials 2009, 27 (2009).

113 Wallentin, J. et al. Single GaInP nanowire pin junctions near the direct to indirect bandgap crossover point. Applied Physics Letters 100, 251103 (2012).

114 Majni, G. \& Ottaviani, G. Large- area uniform growth of $<100>$ Si through Al film by solid epitaxy. Applied Physics Letters 31, 125-126 (1977).

115 Wang, Y., Schmidt, V., Senz, S. \& Gösele, U. Epitaxial growth of silicon nanowires using an aluminium catalyst. Nature nanotechnology 1, 186-189 (2006).

116 Sprokel, G. \& Fairfield, J. Diffusion of gold into silicon crystals. Journal of The Electrochemical Society 112, 200-203 (1965).

117 Bullis, W. Properties of gold in silicon. Solid-State Electronics 9, 143-168 (1966).

118 Perea, D. E. et al. Three-dimensional nanoscale composition mapping of semiconductor nanowires. Nano letters 6, 181-185 (2006).

119 Zhang, S. et al. Relative influence of surface states and bulk impurities on the electrical properties of Ge nanowires. Nano letters 9, 3268-3274 (2009).

120 Dimoulas, A., Tsipas, P., Sotiropoulos, A. \& Evangelou, E. Fermi-level pinning and charge neutrality level in germanium. Applied physics letters 89, 252110-252110 (2006).

$121 \mathrm{McPhail}, \mathrm{D}$. Applications of secondary ion mass spectrometry (SIMS) in materials science. Journal of materials science 41, 873-903 (2006).

122 Putnam, M. C. et al. Secondary ion mass spectrometry of vapor- liquid-solid grown, Au-catalyzed, Si wires. Nano letters 8, 3109-3113 (2008).

123 Kelzenberg, M. D. et al. Photovoltaic measurements in single-nanowire silicon solar cells. Nano letters 8, 710-714 (2008).

124 Boettcher, S. W. et al. Energy-conversion properties of vapor-liquid-solid-grown silicon wire-array photocathodes. Science 327, 185-187 (2010).

125 Allen, J. E. et al. High-resolution detection of Au catalyst atoms in Si nanowires. Nature Nanotechnology 3, 168-173 (2008). 
126 Bar-Sadan, M., Barthel, J., Shtrikman, H. \& Houben, L. Direct imaging of single Au atoms within GaAs nanowires. Nano letters 12, 2352-2356 (2012).

127 Hannon, J., Kodambaka, S., Ross, F. \& Tromp, R. The influence of the surface migration of gold on the growth of silicon nanowires. Nature 440, 69-71 (2006).

128 Stolwijk, N., Schuster, B., Hölzl, J., Mehrer, H. \& Frank, W. Diffusion and solubility of gold in silicon. Physica B+C 116, 335-342 (1983).

129 den Hertog, M. I. et al. Control of gold surface diffusion on Si nanowires. Nano letters $\mathbf{8}$, 1544-1550 (2008).

130 Kawashima, T. et al. Control of surface migration of gold particles on Si nanowires. Nano letters 8, 362-368 (2008).

131 Kodambaka, S., Hannon, J. B., Tromp, R. M. \& Ross, F. M. Control of Si nanowire growth by oxygen. Nano letters 6, 1292-1296 (2006).

132 Dayeh, S. A., Mack, N. H., Huang, J. Y. \& Picraux, S. Advanced core/multishell germanium/silicon nanowire heterostructures: The Au-diffusion bottleneck. Applied Physics Letters 99, 023102 (2011).

133 Dayeh, S., Gin, A. \& Picraux, S. Advanced core/multishell germanium/silicon nanowire heterostructures: Morphology and transport. Applied Physics Letters 98, 163112 (2011).

134 Breuer, S. et al. Suitability of Au-and self-assisted GaAs nanowires for optoelectronic applications. Nano letters 11, 1276-1279 (2011).

135 Jiang, N. et al. Long minority carrier lifetime in Au-catalyzed GaAs/AlxGa1- xAs coreshell nanowires. Applied Physics Letters 101, 023111 (2012).

136 Jiang, N. et al. Enhanced minority carrier lifetimes in GaAs/AlGaAs core-shell nanowires through shell growth optimization. Nano letters 13, 5135-5140 (2013).

137 Nguyen, P., Ng, H. T. \& Meyyappan, M. Catalyst metal selection for synthesis of inorganic nanowires. Advanced Materials 17, 1773-1777 (2005).

138 Bae, J., Kulkarni, N. N., Zhou, J. P., Ekerdt, J. G. \& Shih, C.-K. VLS growth of Si nanocones using Ga and Al catalysts. Journal of Crystal Growth 310, 4407-4411 (2008).

139 Cui, Y., Duan, X., Hu, J. \& Lieber, C. M. Doping and Electrical Transport in Silicon Nanowires. The Journal of Physical Chemistry B 104, 5213-5216, doi:10.1021/ jp0009305 (2000).

140 Duan, X. \& Lieber, C. M. General synthesis of compound semiconductor nanowires. Advanced Materials 12, 298-302 (2000).

141 Dayeh, S. A. et al. High Electron Mobility InAs Nanowire Field- Effect Transistors. small 3, 326-332 (2007).

142 Dayeh, S. A., Susac, D., Kavanagh, K. L., Yu, E. T. \& Wang, D. Field dependent transport properties in InAs nanowire field effect transistors. Nano letters 8, 3114-3119 (2008).

143 Ford, A. C. et al. Diameter-dependent electron mobility of InAs nanowires. Nano Letters 9, 360-365 (2008).

144 Dayeh, S. A., Yu, E. T. \& Wang, D. Transport coefficients of InAs nanowires as a function of diameter. small 5, 77-81 (2009).

145 Dayeh, S. A., Soci, C., Paul, K., Edward, T. Y. \& Wang, D. Influence of surface states on the extraction of transport parameters from InAs nanowire field effect transistors.

Applied Physics Letters 90, 162112 (2007). 
146 Dayeh, S. A., Soci, C., Paul, K., Edward, T. Y. \& Wang, D. Transport properties of InAs nanowire field effect transistors: The effects of surface states. Journal of Vacuum Science \& Technology B 25, 1432-1436 (2007).

147 Storm, K. et al. Spatially resolved Hall effect measurement in a single semiconductor nanowire. Nature nanotechnology 7, 718-722 (2012).

148 DeGrave, J. P., Liang, D. \& Jin, S. A general method to measure the hall effect in nanowires: examples of FeS2 and mnsi. Nano letters 13, 2704-2709 (2013).

149 Lindgren, D. et al. Study of carrier concentration in single InP nanowires by luminescence and Hall measurements. Nanotechnology 26, 045705 (2015).

150 Richter, T., Meijers, H. L. t. R., Calarco, R. \& Marso, M. Doping concentration of GaN nanowires determined by opto-electrical measurements. Nano letters 8, 3056-3059 (2008).

151 Allen, J. E., Perea, D. E., Hemesath, E. R. \& Lauhon, L. J. Nonuniform nanowire doping profiles revealed by quantitative scanning photocurrent microscopy. Advanced Materials 21, 3067-3072 (2009).

152 Schmidt, V. et al. Using the Seebeck coefficient to determine charge carrier concentration, mobility, and relaxation time in InAs nanowires. Applied Physics Letters 104, 012113, doi:doi:http://dx.doi.org/10.1063/1.4858936 (2014).

153 Imamura, G. et al. Distribution of active impurities in single silicon nanowires. Nano letters 8, 2620-2624 (2008).

154 Roddaro, S. et al. InAs nanowire metal-oxide-semiconductor capacitors. Applied Physics Letters 92, 253509, doi:doi:http://dx.doi.org/10.1063/1.2949080 (2008).

155 Ilani, S., Donev, L. A., Kindermann, M. \& McEuen, P. L. Measurement of the quantum capacitance of interacting electrons in carbon nanotubes. Nature Physics 2, 687-691 (2006).

156 Tu, R., Zhang, L., Nishi, Y. \& Dai, H. Measuring the capacitance of individual semiconductor nanowires for carrier mobility assessment. Nano letters 7, 1561-1565 (2007).

157 Garnett, E. C. et al. Dopant profiling and surface analysis of silicon nanowires using capacitance-voltage measurements. Nat Nano 4, 311-314, doi:http://www.nature.com/ nnano/journal/v4/n5/suppinfo/nnano.2009.43 S1.html (2009).

158 Yang, C., Zhong, Z. \& Lieber, C. M. Encoding electronic properties by synthesis of axial modulation-doped silicon nanowires. Science 310, 1304-1307 (2005).

159 Christesen, J. D., Pinion, C. W., Zhang, X., McBride, J. R. \& Cahoon, J. F. Encoding abrupt and uniform dopant profiles in vapor-liquid-solid nanowires by suppressing the reservoir effect of the liquid catalyst. ACS nano 8, 11790-11798 (2014).

160 Cheng, G. et al. Current rectification in a single GaN nanowire with a well-defined $\mathrm{p}-\mathrm{n}$ junction. Applied Physics Letters 83, 1578-1580 (2003).

161 Le, S. T., Jannaty, P., Zaslavsky, A., Dayeh, S. \& Picraux, S. Growth, electrical rectification, and gate control in axial in situ doped pn junction germanium nanowires. Applied Physics Letters 96, 262102 (2010).

162 Mohite, A. et al. Highly efficient charge separation and collection across in situ doped axial VLS-grown Si nanowire p-n junctions. Nano letters 12, 1965-1971 (2012).

163 Arstila, K. et al. Nanoprober-based EBIC measurements for nanowire transistor structures. Microelectronic Engineering 105, 99-102 (2013). 
164 Hoffmann, S. et al. Axial pn junctions realized in silicon nanowires by ion implantation. Nano letters 9, 1341-1344 (2009).

165 Vallett, A. L. et al. Fabrication and characterization of axially doped silicon nanowire tunnel field-effect transistors. Nano letters 10, 4813-4818 (2010).

166 Bassani, F. et al. Dopant profiling in silicon nanowires measured by scanning capacitance microscopy. physica status solidi (RRL)-Rapid Research Letters $\mathbf{8}, 312-316$ (2014).

167 den Hertog, M. I. et al. Mapping active dopants in single silicon nanowires using off-axis electron holography. Nano letters $\mathbf{9}, 3837-3843$ (2009).

$168 \mathrm{Gan}$, Z. et al. Mapping electrostatic profiles across axial pn junctions in Si nanowires using off-axis electron holography. Applied Physics Letters 103, 153108 (2013).

169 Darbandi, A., McNeil, J., Akhtari Zavareh, A., Watkins, S. \& Kavanagh, K. L. Direct Measurement of the Electrical Abruptness of a Nanowire pn Junction. Nano letters (2016).

170 Dalpian, G. M. \& Chelikowsky, J. R. Self-purification in semiconductor nanocrystals. Physical review letters 96, 226802 (2006).

171 Hong, K.-H., Kim, J., Lee, J. H., Shin, J. \& Chung, U.-I. Asymmetric doping in silicon nanostructures: the impact of surface dangling bonds. Nano letters 10, 1671-1676 (2010).

172 Leao, C. R., Fazzio, A. \& da Silva, A. J. Confinement and surface effects in B and P doping of silicon nanowires. Nano letters 8, 1866-1871 (2008).

173 Miranda-Durán, Á., Cartoixà, X., Cruz Irisson, M. \& Rurali, R. Molecular doping and subsurface dopant reactivation in Si nanowires. Nano letters 10, 3590-3595 (2010).

174 Rosini, M. \& Magri, R. Surface effects on the atomic and electronic structure of unpassivated GaAs nanowires. ACS nano 4, 6021-6031 (2010).

175 Shu, H., Chen, X., Ding, Z., Dong, R. \& Lu, W. First-principles study of the doping of InAs nanowires: Role of surface dangling bonds. The Journal of Physical Chemistry $C$ 115, 14449-14454 (2011).

176 Diarra, M., Niquet, Y.-M., Delerue, C. \& Allan, G. Ionization energy of donor and acceptor impurities in semiconductor nanowires: Importance of dielectric confinement. Physical Review B 75, 045301 (2007).

177 Björk, M. T., Schmid, H., Knoch, J., Riel, H. \& Riess, W. Donor deactivation in silicon nanostructures. Nature Nanotechnology 4, 103-107 (2009).

178 Khanal, D., Yim, J. W., Walukiewicz, W. \& Wu, J. Effects of quantum confinement on the doping limit of semiconductor nanowires. Nano letters 7, 1186-1190 (2007).

179 Walukiewicz, W. Intrinsic limitations to the doping of wide-gap semiconductors. Physica B: Condensed Matter 302, 123-134 (2001).

180 Hilse, M., Ramsteiner, M., Breuer, S., Geelhaar, L. \& Riechert, H. Incorporation of the dopants Si and Be into GaAs nanowires. Applied Physics Letters 96, 193104 (2010).

181 Ketterer, B., Mikheev, E., Uccelli, E. \& Fontcuberta i Morral, A. Compensation mechanism in silicon-doped gallium arsenide nanowires. Applied Physics Letters 97, 223103 (2010).

182 Perea, D. E., Li, N., Dickerson, R. M., Misra, A. \& Picraux, S. Controlling heterojunction abruptness in VLS-grown semiconductor nanowires via in situ catalyst alloying. Nano letters 11, 3117-3122 (2011). 